\title{
ANÁLISE DE EXPERIMENTOS EM FAIXAS COM SUBPARCELAS SUBDIVIDIDAS
}

\author{
ELIZA HELENA DE SOUZA FARIA \\ Engenheira-Agrônoma
}

Orientador: Prof. Dr. HUMBERTO DE CAMPOS

\begin{abstract}
Dissertação apresentada à Escola Superior de Agricultura "Luiz de Queiroz", da Universidade de São Paulo, para obtenção do título de Mestre em Agronomia. Área de Concentração: Estatística $\theta$ Experimentação Agronômica.
\end{abstract}

PIRACICABA

Estado de São Paulo - Brasil

Abril, 1984 
Aos meus pais,

que sempre fizeram de suas vidas

um exemplo para a minha;

Aos meus irmãos,

pelo carinho e dedicação;

A. Elzinha e Oswaldo,

pelo amor que sempre nos uniu,

Ao Märio,

D E D I C 0. 


\section{AGRADECIMENTOS}

Ao Dr. Humberto de Campos, Professor Titular do Departamento de Matemätica e Estatística da ESALQ/USP, pela orientação segura e amiga na execução deste trabalho.

Aos Professores do Curso de Pós-Graduação em Estatística e Experimentação Agronōmica da ESALQ/USP, pelos ensinamentos recebidos.

Ao Professor Dr. Antonio Francisco Iemma, que esteve sem pre presente com sua solidariedade e ajuda durante a rea1ização do Curso.

Aos Professores Décio Barbin e Clóvis Pompilio de Abreu, por sua acolhida, amizade e ensinamentos.

Aos Professores do Departamento de Matemätica, à Diretoria do Instituto de Ciēncias Exatas, e à Reitoria da Universidade Federal Rural do Rio de Janeiro, pelo afasta mento concedido.

A CAPES - Coordenação de Aperfeiçoamento do Pessoal do Nível Superior, pela bolsa de estudos concedida.

Aos amigos Élio Paulo Zonta, Margareth C. Toyama Udo e Agós tinho Roberto de Abreu, pela amizade e troca de idéias durante o Curso. 
Aos colegas do Curso de Pós-Graduação em Estatística e Ex perimentação Agronômica, pelo excelente convívio.

Aos Funcionários do Departamento de Matemática e Estatistica da ESALQ/USP, pela atenção e amizade.

A Srta. Maria Izalina Ferreira Alves, pela amizade e pe los serviços datilográficos.

A todos que, direta ou indiretamente, colaboraram para a realização deste trabalho. 


\section{I $N$ D}

Pag.

RE SUMO

vii

SUMMARY

$i x$

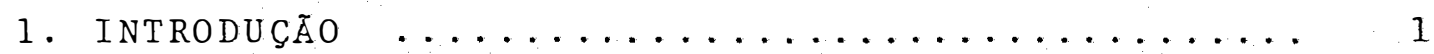

2. REVISÃO BIBLIOGRÁFICA $\ldots \ldots \ldots \ldots \ldots \ldots \ldots \ldots$

$2.1-\operatorname{Introdução~} \ldots \ldots \ldots \ldots \ldots \ldots \ldots \ldots \ldots \ldots \ldots \ldots \ldots \ldots$

2.2 - Modelo Matemätico $\ldots \ldots \ldots \ldots \ldots \ldots$

2.3 - Anālise de Variāncia $\ldots \ldots \ldots \ldots \ldots \ldots \ldots$

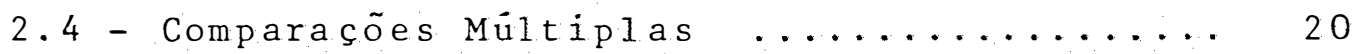

3. Desenvolvimento TeOrico $\ldots \ldots \ldots \ldots \ldots \ldots \ldots \ldots$

3.1 - Modelo Matemático ................. 23

3.2 - Sistema de Equações Normais ......... 25

3.2.1 - obtenção do sistema de equações nor

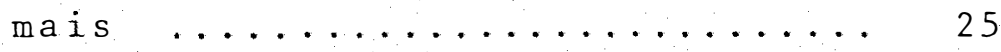

3.2 .2 - Solução do sistema $\ldots \ldots \ldots \ldots \ldots 32$

3.3 - Anāilse de Variāncia .............. 35

3.3.1 - Obtenção das somas de quadrados . . 35

3.3.2 - Esperança matemática dos quadrados médios $\ldots \ldots \ldots \ldots \ldots \ldots \ldots \ldots$

3.3.3 - Testes de hipöteses e quadro de anàlise de variāncia .......... 52 
Pàg.

3.4 - Variāncias de Contrastes .......... 56

3.5 - Comparações Mültiplas $\ldots \ldots \ldots \ldots \ldots$

4. UM EXEMPLO DE APLICAÇ̃̃O ................. 74

5. CONCLUSOES $\ldots \ldots \ldots \ldots \ldots \ldots \ldots \ldots \ldots \ldots \ldots \ldots \ldots \ldots \ldots \ldots \ldots \ldots \ldots$

6. REFERENCIAS BIbLIOGRÁfICAS $\ldots \ldots \ldots \ldots \ldots \ldots$ 


\title{
ANALISE DE EXPERIMENTOS EM FAIXAS COM SUBPARCELAS SUBDIVIDIDAS
}

\author{
Autor: $\quad$ Eliza Helena de Souza Faria \\ Orientador: Prof. Dr. Humberto de Campos
}

R E S U M O

o presente estudo teve como objetivo deduzir. os fundamentos da anảlise de variância e dos métodos de comparações mültiplas nos experimentos em fáixas com as subparcelas subdivididas.

$$
\begin{aligned}
& \text { Para tanto adotou-se o modelo linear: } \\
& y_{i k \ell j}=\mu+t_{i}+b_{j}+(t b)_{i j}+t_{k}^{\prime}+\left(t^{\prime} b\right)_{k j}+t t_{i k}^{\prime}+
\end{aligned}
$$

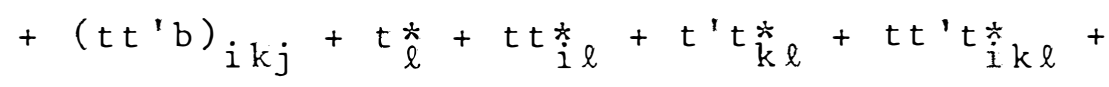

$$
\begin{aligned}
& +e_{i k \ell j,},
\end{aligned}
$$

onde os parâmetros portadores do indice j foram considera dos aleatórios.

Foram deduzidas as somas de quadrados, as es

peranças dos quadrados médios e obtidos os critérios para os testes das hipóteses inerentes à análise de variāncia 
viii.

e para as comparações mültiplas.

Um exemplo ilustrativo, com dados de produção de cana-de-açücar, é apresentado.

Os resultados obtidos permitiram concluir pe 1 a analogia do experimento em questão, ora com os experimentos em faixas com très residuos, ora com os experimenLos em parcelas sub-subdivididas. 
ANALYSIS OF EXPERIMENTS IN STRIPS WITH SPLIT SUB-PLOT

\begin{abstract}
Author:
Eliza Helena de Souza Faria
\end{abstract}

Adviser:

Prof. Dr. Humberto de Campos

The present study objetives the development of the fundamentals of the variance analysis and the multiple comparisons methods in experiments in strips with split-sub 1ot:

The following linear model was adopted:

$$
\begin{aligned}
y_{i k l j}=\mu & +t_{i}+b_{j}+(t b)_{i j}+t_{k}^{\prime}+\left(t^{\prime} b\right)_{k j}+t_{i k}^{\prime}+ \\
& +\left(t t^{\prime} b\right)_{i k j}+t_{\ell}^{*}+t t_{i \ell}^{*}+t^{\prime} t_{k \ell}^{*}+t t^{\prime} t_{i k \ell}^{+}+ \\
& +e_{i k \ell j}
\end{aligned}
$$

Where the parameters carrying the $j$ index were considered r andom .

$$
\text { Sum of squares and expected mean squares, and }
$$

criteria for the usual hypotheses tests deduced from the Analysis of Variance table and from the multiple comparisons were obtained. 
The results implied the analogy of the present experiment with either the 3 residual strip experiments or with the usual split-plit-plot experiments. 


\section{INTRODUCAOO}

Em experimentos agricolas de campo, conduzidos para estudar simultaneamente os efeitos da variação de mais de um fator, os pesquisadores podem encontrar dificuldades präticas para casualizar completamente todos os tratamentos dentro de um mesmo bloco.

Para sanar essa dificuldade operacional, pode-se lançar mão dos experimentos em faixas.

Assim, por exemplo, quando se quer considerar no mesmo experimento dois tipos de tratamentos (A e B), que requerem parcelas relativamente grandes, se estabelecem em cada bloco os niveis de um dos tratamentos dis tribuidos ao acaso em faixas paralelas e os niveis do outro tratamento em faixas perpendiculares às anteriores, distribuindo-os tambëm ao acaso. 
Em cada nivel de A tem-se os níveis de B como subparcelas e vice-versa. Assim sendo, tanto os níveis de A como os de B são considerados parcelas principais, e $\underline{n}$ quanto que a interação $A \times B$ em cada um dos seus níveis $A_{i} \quad B_{j}$ constitui as subparcelas.

As subparcelas formadas pela interseção das Faixas podem ser subdivididas para acomodar um terceiro grupo de tratamentos, ocasionando um quarto resíduo.

Várias situações podem ainda ser caracterizadas mostrando que a melhor solução prática converge pa ra os experimentos em faixas. Assim, por exemplo, em experimentos com épocas de colheita, quando a mesma é feita por meios mecānicos; ou em aplicação mecânica de adubos, corretivos, inseticidas; em experimentos que envolvem tipos de preparo de solo, profundidade de aração; ex perimentos de manejo em pastagens; etc.

Por outro lado, apesar da grande aplicabili dade dos experimentos em faixas, sentiu-se na revisão bi bliogräfica uma lacuna no tocante às justificativas teóricas das förmulas encontradas, quadros de anälise de va riância e comparaçóes mültiplas.

Alèm disso, constatou-se que os textos tradicionais de Estatistica Experimental e mesmo os artigos mais recentes, praticamente não fornecem subsídios para 
a anālise de experimentos em faixas com subparcelas subdi vididas. Exceção deve ser feita, no entanto, para um arti go de HEDAYETULLAH et alii (1944), que apresenta um quadro de anālise de variāncia para ensaios em faixas com trés tratamentos, mas sem apresentar quaisquer considerações teóricas sobre sua obtenção.

Desse modo, motivados pela necessidade da de dução de tais anālises, descortinou-se como um dos objeti vos desta pesquisa, a proposta de um modelo, bem como o estudo completo dos critérios para os testes de hipóteses de interesse nos experimentos em faixas com as subparce1 as subdivididas, em que se tem I tratamentos $T$ e $K$ trata-. mentos $T^{\prime}$ dispostos nas parcelas principais em faixas; L tratamentos $\mathrm{T}^{*}$ dispostos em cada subparcela $\mathrm{TT}^{\prime}$ e J blo$\cos$. 


\title{
2. REVISÃO BIBLIOGRĀFICA
}

\section{1 - Introdução}

\begin{abstract}
De modo geral, os autores tèm sido unānimes em ressaltar a utilidade dos experimentos em faixas, para o caso em que se deseja avaliar dois tipos de tratanentos dispostos em parcelas relativamente grandes.

Para FEDERER（1955），LOMA（1966） e PIMENTEL GOMES (1976), dentre outros, a lém do tamanho das parcelas, outros fatores associados direta ou indiretamente ao experimento tais como processos de mecanização, níveis de irrigação, etc., podem nos levar à utilização dos experimentos em faixas.

Por outro lado, FEDERER (1955) aconselha o uso dos experimentos em faixas, quando os efeitos indivi
\end{abstract}


duais dos dois tipos de tratamentos podem ser relativamen te pequenos em relação ao efeito da interação entre eles. Por sua vez, LOMA (1966) concorda com KEMPTHORNE (1952) e FEDERER (1955), dentre outros, ao mostrar as vantagens desses experimentos no tocante a sua casuali zação, comparativamente a outros delineamentos.

Assim, para um ensaio com dois tratamentos A e B, em dois e trés níveis respectivamente, cada bloco po deria ser dividido em faixas horizontais, $A_{i}$, e em faixas verticais, $B_{j}$, resultando para um bloco qualquer, por exem plo:

\begin{tabular}{|c|c|c|c|}
\hline & $\mathrm{B}_{3}$ & $B_{1}$ & $\mathrm{~B}_{2}$ \\
\hline $\mathrm{A}_{2}$ & $\mathrm{~A}_{2} \mathrm{~B}_{3}$ & $\mathrm{~A}_{2} \mathrm{~B}_{1}$ & $\mathrm{~A}_{2} \mathrm{~B}_{2}$ \\
\hline $\mathrm{A}_{1}$ & $\mathrm{~A}_{1} \mathrm{~B}_{3}$ & $\mathrm{~A}_{1} \mathrm{~B}_{1}$ & $\mathrm{~A}_{1} \mathrm{~B}_{2}$ \\
\hline
\end{tabular}

e de modo anälogo para os demais blocos do ensaio, que apresentariam diferenças apenas quanto à casualização. Em cada repetição são estabelecidos os niveis de um dos tratamentos, distribuidos ao acaso em faixas paralelas e os níveis do outro tratamento em faixas perpendiculares às anteriores, distribuindo-os tambëm ao acaso em cada repetição, facilitando sobremaneira, a instalação dos experimentos de campo. 
FEDERER (1955) cita as seguintes vantagens

dos ensaios em faixas:

a) as subparcelas podem ser relativamente pequenas, enquanto que as parcelas podem ter grandes dimensões;

b) maior precisão na informação para a interação dupla dos tratamentos que estão em faixas;

e suas principais desvantagens :

a) as informações sobre os tratamentos que estão em faixas são menos precisas que aquelas obtidas em blocos casualizados;

b) a análise estatistica é mais complexa do que nos delineamentos em blocos casualizados, principalmente para situações não ortogonais e anālise de covariāncia.

\section{2 - Modelo matemātico}

Segundo KEMPTHORNE (1952), o modelo adequado para ensaios em faixas, com dois tratamentos, é o modelo linear do tipo misto:

$$
\begin{aligned}
y_{i j k}=\mu & +r_{i}+a_{j}+(r a)_{i j}+b_{k}+(r b)_{i k}+(a b)_{j k}+ \\
& +(r a b)_{i j k},
\end{aligned}
$$


onde: $y_{i j k}=$ valor da parcela que recebeu o k-ésimo tratamento $B$ e o j-ésimo tratamento A na i- èsima repetição;

$\mu=$ mèdia geral

$r_{i}=$ efeito da i-èsima repetição;

$a_{j}=$ efeito do j-ésimo tratamento A;

$(r a)_{i j}=$ efeito da interação entre a i-ésima repetição e o j-ésimo tratamento A;

$\mathrm{b}_{\mathrm{k}}=$ efeito do k-ésimo tratamento B;

$(r b)_{i k}=$ efeito da interação entre a i-ésima repetição e o k-ésimo tratamento B;

$(a b)_{j k}=$ efeito da interação entre os tratamentos A e B ;

$(r a b)_{i j k}=$ erro experimental

onde, $(r a)_{i j},(r b)_{i k} e(r a b)_{i j k}$ são considerados erros ex perimentais para os tratamentos A, B e a interaça $A \times B$, normal e independentemente distribuidos com média zero e variāncias $\sigma_{\mathrm{r}}^{2}, \sigma_{\mathrm{C}}^{2}$ e $\sigma_{S}^{2}$, respectivamente.

CHAKRABARTI ( 1962 ) apresenta para experimen tos com parcelas subdivididas com as subparcelas em faixas, o seguinte modelo:

$$
y_{i j k}=\mu+\alpha_{i}+\gamma_{j}+\tau_{k}+\delta_{j k}+\varepsilon_{i j k},
$$

para $i=1,2, \ldots, r ; j=1,2, \ldots, \alpha ; k=1,2, \ldots, \beta$; 
onde: $y_{i j k}=$ valor observado da parcela que recebeu o k-èsimo sub-tratamento e j-ésimo tratamento principal na i-ésima repetição;

$$
\mu=\text { média gera } 1 \text {; }
$$

$\alpha_{i}=$ efeito do i-ésimo bloco;

$\gamma_{j}=$ efeito do j-ésimo tratamento $Y$;

$\tau_{k}=$ efeito do k-ésimo tratamento $\tau$;

$\delta_{j k}=$ efeito da interação da i-ésima repetição com

o k-èsimo sub-tratamento;

$\varepsilon_{i j k}=$ erro experimental

Para o autor, as observações $y_{i j k}, y_{i j k} e y_{i j k}$, $y_{i j k}$, são correlacionadas, resultando:

$$
E\left(\varepsilon_{i j k}\right)=0 \quad, \quad V\left(\varepsilon_{i . j k}\right)=\sigma^{2} \quad e
$$

$$
\operatorname{cov}\left(\varepsilon_{i j k}, \varepsilon_{i} \prime^{\prime}{ }^{\prime} k^{\prime}\right)= \begin{cases}\rho_{1} \sigma^{2} & \text { se } i=i^{\prime}, j=j^{\prime}, k=k^{\prime} \\ \rho_{2} \sigma^{2} & \text { se } i=i^{\prime}, j \neq j^{\prime}, k=k^{\prime} \\ 0 & \text { em outros casos. }\end{cases}
$$

ZIMMERMANN (1973), para a aplicação dos më-odos em estudo, usa um ensaio de adubação e calagem, em milho (Zea mays, L.) conduzido no Parque Estadual de Vila Velha, PR, no periodo de 1963/64 a 1970/71. Foi em pregado o del ineamento em faixas, em blocos casualizados, 
com duas repetições, sendo aplicadosàs parcelas (horizontais) os diferentes niveis de calcário, e as faixas (parcelas verticais) os niveis de adubação completa NPK. O mo delo matemático para cada período foi:

$$
y_{i j k}=m+\rho_{j}+a_{i}+\alpha_{i j}+b_{k}+\beta_{j k}+(a b)_{i k}+\varepsilon_{i j k}(2 \cdot 2 \cdot 3)
$$

onde: $\quad y_{i j k}=$ observação da ik-èsima parcela no j-èsimo blo co;

$$
\begin{aligned}
\mathrm{m}= & \text { média geral da população; } \\
\rho_{j}= & \text { efeito aleatório do j-ésimo bloco; } \\
a_{i}= & \text { efeito do i-ésimo nível do tratamento A; } \\
\alpha_{i j}= & \text { efeito aleatório associado à ij-ésima obser } \\
& \text { vação; } \\
b_{k}= & \text { efeito do k-ésimo nível do trátamento B; } \\
B_{j k}= & \text { efeito aleatório associado à kj-ésima obser } \\
& \text { vação; } \\
(a b)_{i k}= & \text { efeito interado do i-ēsimo nivel do trata- } \\
& \text { mento A como k-ésimo nivel do tratamento B; } \\
\varepsilon_{i j k}= & \text { erro experimental. }
\end{aligned}
$$

Além disso, ZIMMERMANN (1973) apresenta o se guinte modelo para anälise de um experimento de adubaça . e calagem em milho, executado num delineamento em faixas, durante oito anos, em um mesmo local: 


$$
\begin{aligned}
y_{i j k m}=m & +\rho_{j}+a_{i}+\alpha_{i j}+c_{m}+(\rho c)_{j m}+(a c)_{i m}+ \\
& +\beta_{i j m}+b_{k}+\gamma_{j k}+(b c)_{k m}+\delta_{j k m}+(a b)_{i k}+ \\
& +\theta_{i j k}+(a b c)_{i k m}+\varepsilon_{i j k m},
\end{aligned}
$$

onde: $y_{i j k m}=$ produção da ijk-ésima parcela, no m-ésimo ano;

$\mathrm{m}=$ média geral da população;

$\rho_{j}=$ efeito aleatório do j-ésimo bloco;

$a_{i}=$ efeito do i-ésimo nivel do tratamento A;

$\alpha_{i j}=$ efeito aleatório associado à ij-ésima obser vação;

$c_{m}=$ efeito do m-ësimo ano;

$(\rho c)_{j m}=$ efeito do j-ésimo bloco, no m-ésimo ano;

$(\mathrm{ac})_{i m}=$ efeito interado do i-ésimo nível do tratamento A com o m-ésimo ano;

$\beta_{i j m}=$ efeito aleatório da ijm-ésima observação;

$\mathrm{b}_{\mathrm{k}}=$ efeito do k-ésimo nivel do tratamento B;

$\gamma_{j k}=$ efeito aleatório associado a jk-ésima obser vação;

$(b c)_{k m}=$ efeito interado do k-ésimo nivel do tratamento B com o m-ésimo ano;

$\delta_{j k m}=$ efeito aleatório, associado à jkm-ésima obs ervação ;

$(a b)_{i k}=$ efeito interado do i-ésimo nível do tratamento A com o k-ésimo nivel do tratamento B; 


$$
\begin{aligned}
\theta_{i j k}= & \text { efeito aleatório associado à interaça do i- } \\
& \text {-ésimo nível de A com o k-èsimo nivel do tratá } \\
& \text { mento B, no j-ésimo bloco; }
\end{aligned}
$$

$(a b c)_{i k m}=$ efeito interado do i-ésimo nivel do tratamento A com o k-ésimo nivel do tratamento B, nom-és mo a no;

$$
\varepsilon_{i j k m}=\text { efeito aleatório ou influéncia do acaso. }
$$

$$
\text { O modelo matemático é do tipo misto, e consi }
$$
deram-se aleatörios:

$$
\rho_{j}, \alpha_{i j}, \beta_{i j k}, \gamma_{j k}, \delta_{j k m}, \theta_{i j k},(\rho c)_{j m} e \varepsilon_{i j k m},
$$

com médias zero e variāncias

$$
\sigma_{\rho}^{2}, \sigma_{\alpha}^{2}, \sigma_{\beta}^{2}, \sigma_{\gamma}^{2}, \sigma_{\delta}^{2}, \sigma_{\theta}^{2}, \sigma_{\rho c}^{2} \mathrm{e} \sigma^{2},
$$

respectivamente.

\section{3 - Anālise de Variāncia}

$\mathrm{Na}$ revisão dos textos existentes na área, no tou-se também, que os autores apresentam os experimentos em faixas como uma extensão dos experimentos fatoriais, notadamente FEDERER (1955) e LOMA (1966), ou como uma extensão dos experimentos com parcelas subdivididas, como GOULdEN (1939), KEMPTHORNe (1952) e PIMENTEL GOMES (1976). Desse modo, esses autores não demonstraramas 
somas de quadrados, suas esperanças matemáticas e os prin cipais testes de hipöteses. Exceção deve ser feita, no en tanto, para CHAKRABARTI (1962) e ZIMMERMANN (1973), aquele usando dois tratamentos e este estudando a análise con junta de experimentos em. faixas.

Assim, como abordado anteriormente, apenas em HEDAYETULLAH et alii (1944) encontrou-se um quadro de anä lise de variāncia para ensaios em faixas incluindo trés tipos de tratamentos, mas sem quaisquer considerações teó ricas sobre sua obtenção.

NAIR (1944) considera um experimento de dois fatores com $p$ níveis $a_{1}, a_{2}, \ldots, a_{p}$ de um fator A e q $\underline{n}$ i. veis $b_{1}, b_{2}, \ldots, b_{q}$ de um fator $B$ em todas as combinacões, cada um repetido em r blocos. Cada bloco é dividido em p faixas de coluna, às quais são designados $a_{1}, a_{2}, \ldots, a_{p}$, aleatoriamente, e dividido em q faixas de linhas, às quais são designadas $b_{1}, b_{2}, \ldots, b_{q}$, aleatoriamente. Notarse que não há casualização livre para cada combinação de tra tamento $a_{i} b_{j}$ dentro de cada bloco.

A estrutura da análise da variāncia é mostra da no quadro 1 . 


\begin{tabular}{lc}
$\begin{array}{l}\text { Quadro } 1-\text { Anälise de variáncia para experimentos em fai- } \\
\text { xas, segundo NAIR }(1944) .\end{array}$ & G.L. \\
\hline Causas da Variação & $r-1$ \\
\hline Blocos & $p-1$ \\
Efeito A & $(p-1)(r-1)$ \\
Erro (1) & $q-1$ \\
Efeito B & $(q-1)(r-1)$ \\
Erro (2) & $(p-1)(q-1)$ \\
Interação A x B & $(p-1)(q-1)(r-1)$ \\
Erro (3) & $p q r-1$
\end{tabular}

Para KEMPTHORNE (1952), que considera apenas dois tipos de tratamentos, a anälise de variāncia para ex perimentos em faixas com base no modelo citado em (2.2.1), pode ser efetuada conforme o quadro 2 . 
Quadro 2 - Análise de variāncia para experimentos em faixas, segundo KEMPTHORNE (1952).

\begin{tabular}{|c|c|c|}
\hline Causas da Varia ção & G.L. & $E(Q \cdot M:)$ \\
\hline Repetições $\mathrm{R}$ & $r-1$ & - \\
\hline Tratamentos A & $a-1$ & $\sigma_{s}^{2}+b \sigma_{r}^{2}+-\frac{r b}{a-1} \sum_{j} t_{j}^{2}$ \\
\hline $\mathrm{R} \times \mathrm{A}$ & $(r-1)(a-1)$ & $\sigma_{S}^{2}+b \sigma_{r}^{2}$ \\
\hline Tratamento B & $(b-1)$ & $\sigma_{s}^{2}+a \sigma_{c}^{2}+\frac{r a}{b-1} \sum s_{k}^{2}$ \\
\hline $\mathrm{R} \times \mathrm{B}$ & $(r-1)(b-1)$ & $\sigma_{s}^{2}+: a \sigma_{c}^{2}$ \\
\hline \multirow[t]{2}{*}{ Interação $A \times B$} & \multirow[t]{2}{*}{$(a-1)(b-1)$} & $\sigma^{2}+$ \\
\hline & & $s \quad(a-1)(b-1) j k$ \\
\hline $\mathrm{R} \times \mathrm{A} \times \mathrm{B}$ & $(\mathrm{r}-1)(\mathrm{a}-1)(\mathrm{b}-1)$ & $\sigma_{s}^{2}$ \\
\hline TOTAL & $\mathrm{rab}-1$ & \\
\hline
\end{tabular}

FEDERER (1955), com base no seguinte delinea mento:

REPETIÇAO I

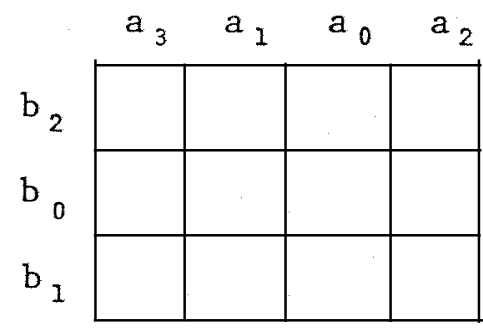

REPETI ÇÃO II

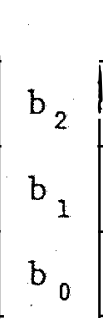

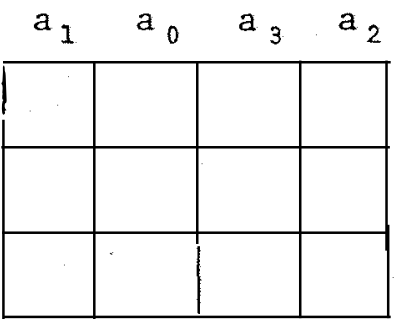

REPET I ÇÃO I I I

\begin{tabular}{|l|l|l|l|}
\multicolumn{1}{c}{$a_{2}$} & $a_{0}$ & $a_{3}$ & $a_{1}$ \\
\hline & & & \\
\hline & & & \\
\hline & & & \\
\hline
\end{tabular}


embora sem apresentar referēncias sobre o modelo matemáti co, propõe a decomposição que consta do quadro 3 .

Quadro 3 - Anālise de variāncia para experimentos em faixas, segundo FEDERER ( 1955 ).

\begin{tabular}{lc}
\hline Causas da Variação & G. L. \\
\hline Repetição & 3 \\
A & 6 \\
Repetição x A $=\operatorname{erro}($ a $)$ & 2 \\
B & 4 \\
Repetição x $B=\operatorname{erro}($ b $)$ & 6 \\
A x B & 12 \\
Erro (c) & 35 \\
\hline TotaL &
\end{tabular}

De modo anälogo, sem apresentar deduções, LOMA (1966), PANSE e SUKHATME (1963), COCHRAN e COX (1976), adotam a decomposição abordada no quadro 2 .

Para CHAKRABART I (1962), que considera um ensaio em "split-plot" com subtratamentos em faixas, a anālise de variância baseada no modelo citado em (2.2.2), pode ser efetuada conforme o quadro 4 . 
Quadro 4 - Anālise de variāncia para experimentos em faixa, segundo CHAKRABARTI (1962).

\begin{tabular}{|c|c|c|}
\hline Causas da Variação & G.L. & S.Q. \\
\hline Tratamento principal & $(\alpha-1)$ & $r \beta \Sigma(y, j .-y . . .)^{2}$ \\
\hline Erro (a) & $(r-1)(\alpha-1)$ & $B \sum \Sigma\left(y_{i j} \cdot y_{i . .}-y_{. j}+y_{. .}\right)^{2}$ \\
\hline Subtratamentos & $(\beta-1)$ & $r \alpha \Sigma\left(y \ldots k^{-y} \ldots\right)^{2}$ \\
\hline Erro（b) & $(r-1)(\beta-1)$ & $\alpha \Sigma \Sigma\left(y_{i . k}{ }^{-y_{i} . .}{ }^{-y} \ldots k^{+y} \ldots\right)^{2}$ \\
\hline Int eração & $(\alpha-1)(\beta-1)$ & $r \Sigma \Sigma\left(y_{\cdot j k^{-y}} \cdot k^{-y} \cdot j \cdot+y . .\right)^{2}$ \\
\hline Erro (c) & $(r-1)(\alpha-1)(\beta-1)$ & $\Sigma \Sigma \Sigma\left(\begin{array}{c}y_{i j k^{-y}} \cdot k^{-y} \cdot j k^{-y} \ldots k^{+y} \cdot j \cdot+ \\
+y \cdot \ldots-y \cdot \ldots\end{array}\right)$ \\
\hline
\end{tabular}

ZIMMERMANN (1973), com base nos modelos citados em 2.2 .3 e 2.2.4, propõe os esquemas de anālise de variāncia, que constam dos quadros 5 e 6 .

Quadro 5 - Anālise de variância para cada período, segundo ZIMMERMANN (1973).

\begin{tabular}{|c|c|c|}
\hline Causas da Variação & G.L. & $E(Q . M)$. \\
\hline Repetições & $\mathrm{J}-1$ & $\sigma^{2}+\mathrm{K} \sigma_{\alpha}^{2}+I \sigma_{\beta}^{2}+I K \sigma_{0}^{2}$ \\
\hline Tratamento A & $\mathrm{I}-1$ & $\sigma^{2}+K \sigma_{\alpha}^{2}+\frac{J K}{I-1} \sum_{i} a_{i}^{2}$ \\
\hline Resíduo (a) & $(I-1)(J-1)$ & $\sigma^{2}+K \sigma_{\alpha}^{2}$ \\
\hline Tratamento $\mathrm{B}$ & $\mathrm{K}-1$ & $\sigma^{2}+I \sigma_{\beta}^{2}+\frac{I J}{K-1} \sum_{k} b_{k}^{2}$ \\
\hline Resíduo (b) & $(\mathrm{J}-1)(\mathrm{K}-1)$ & $\sigma^{2}+I \sigma_{R}^{2}$ \\
\hline$A \times B$ & $(\mathrm{I}-1)(\mathrm{K}-1)$ & $\sigma^{2}+\frac{J}{(I-I)(J-1)} i \sum_{k}(a b) \underset{i k}{2}$ \\
\hline Resíduo (c) & $(\mathrm{I}-1)(\mathrm{J}-1)(\mathrm{K}-1)$ & $\sigma^{2}$ \\
\hline TOTAL & IJK-1 & \\
\hline
\end{tabular}


Quadro 6 - Anālise de variāncia conjunta para o delineamento em faixas durante $m$ anos no mesmo local, segundo ZIMMERMANN (1973).

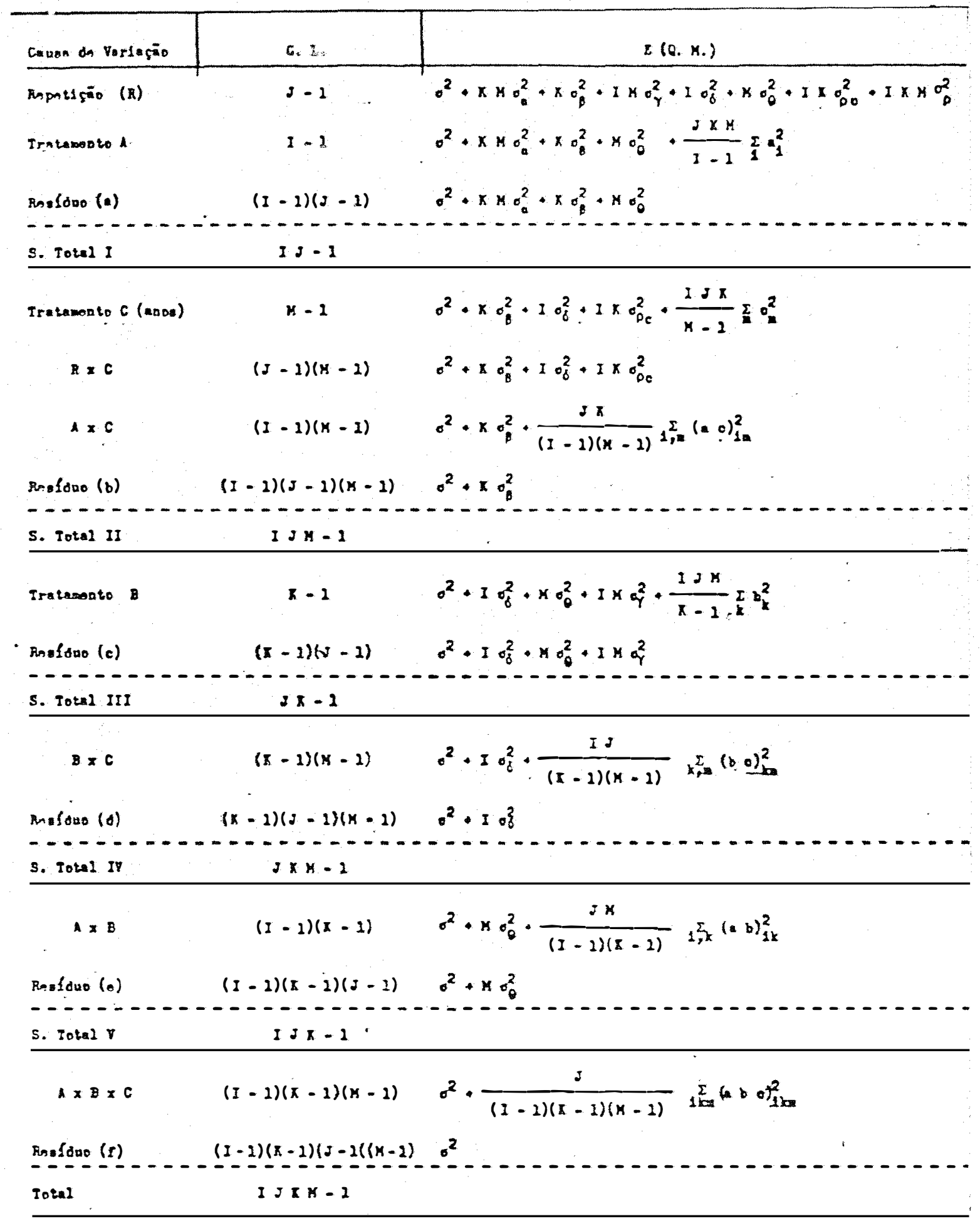


Com a finalidade de se obter uma idéia precí sa dos efeitos dos fatores culturais, tais como época de plantio, idade das mudas, espaçamento, nümero de mudas por cova, etc., sobre a produtividade de algumas linhagens se lecionadas de arroz de diferentes hābitos de maturaçãosob as condições existentes na "Dacca Farm", foi estabelecida uma série de experimentos em 1936-37, e que foram repetidos durante diversos anos sucessivos. HEDAYETULLAH et ali $i$ (1944) tratam dos resultados de um desses experimentos que foi conduzido em 5 anos sucessivos, envolvendo trēs fatores a saber: data de plantio, linhagem de arroz e espaçameñto. Assim, para trēs linhagens de arroz, seis datas de. plantio e trés espaçamentos, com 3 repetições, os autores apresentam o esquema de anälise que consta do quadro 7 acrescentando, assim, um quarto residuo, aos casos mencionados anteriormente. 


\begin{tabular}{|c|c|}
\hline Causas da Variação & G. L. \\
\hline B locos & 2 \\
\hline Linhagens & 2 \\
\hline Erro I & 4 \\
\hline Épocas de plantio & 5 \\
\hline Erro I I & 10 \\
\hline Linhagens $x$ Épocas & 10 \\
\hline Erro I I I & 20 \\
\hline Espaçamentos & 2 \\
\hline Espaçamentos $x$ Épocas & 10 \\
\hline Espaçamentos $x$ Linhagens & 4 \\
\hline Espaçamentos $x$ Épocas $x$ Linhagens & 20 \\
\hline Erro IV & 72 \\
\hline TOTAL & 161 \\
\hline
\end{tabular}




\section{4 - Comparacões Mūltiplas}

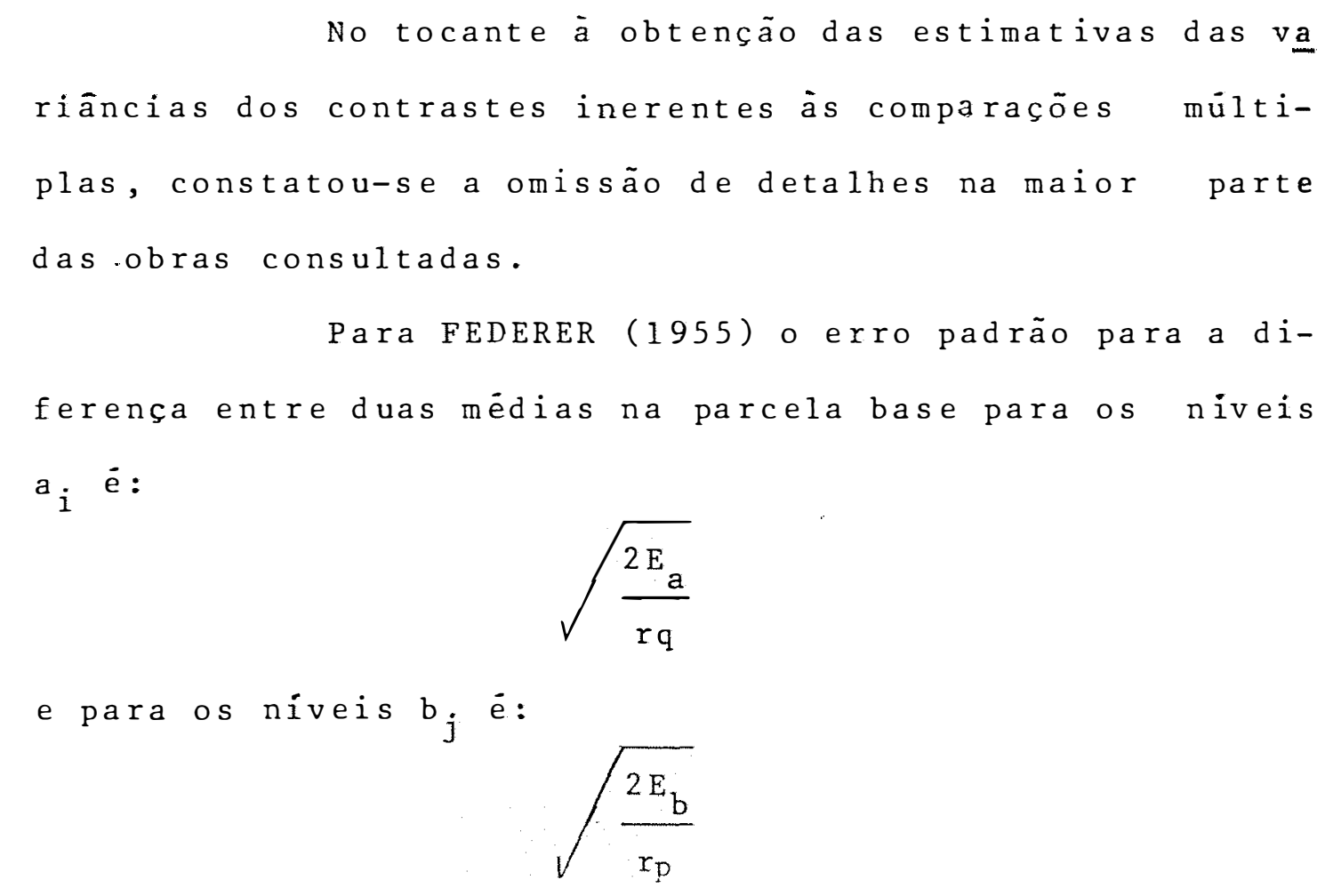

onde: $r=$ nümero de repetições;

$$
\begin{aligned}
& \mathrm{q}=\text { nümero de niveis do fator b; } \\
& \mathrm{p}=\text { número de niveis do fator a. }
\end{aligned}
$$

CHAKRABARTI (1962), considerando o modelo citado em (2.2.2), apresenta as seguintes variāncias de con trastes:

$$
\begin{aligned}
& V\left(y . j .-y . j{ }^{\prime}\right)=\frac{2}{r \beta} \sigma_{1}^{2} \\
& V\left(y \ldots k-y \ldots k^{\prime}\right)=\frac{2}{r \alpha} \sigma_{2}^{2}
\end{aligned}
$$




$$
\begin{aligned}
& V\left(y \cdot j k-y \cdot j{ }^{\prime}\right)=\frac{2}{r \beta}\left[(\beta-1) \sigma_{3}^{2}+\sigma_{1}^{2}\right] \\
& V\left(y \cdot j k-y \cdot j k^{\prime}\right)=\frac{2}{r \alpha}\left[(\beta-1) \sigma_{3}^{2}+\sigma_{2}^{2}\right]
\end{aligned}
$$

Resultados semelhantes podem ser observados em COCHRAN e cox (1976), embora sem referēncias à existēn cia ou não de correlações.

Assim, para $\alpha=$ nümero de niveis de $A, \beta=$ nümero de niveis de $B, r=$ nümero de repetições, COCHRAN e COX (1976) apresentam fórmulas para estimativas do erro padrão da diferença entre duas médias, para os seguintes casos:

a) Diferença entre duas médias de tratamentos A:

$$
\widehat{Y}_{i}=a_{1}-a_{0} \quad \hat{v}\left(\bar{Y}_{i}\right)=\frac{2 E_{a}}{r B} .
$$

b) Diferença entre duas médias de tratamentos B:

$$
\hat{\mathrm{Y}}_{\mathrm{k}}=\mathrm{b}_{\mathrm{l}}-\mathrm{b}_{0} \quad \overrightarrow{\mathrm{V}\left(\hat{\mathrm{Y}}_{\mathrm{k}}\right)=\frac{2 \mathrm{E}_{\mathrm{b}}}{\mathrm{r \alpha}}}
$$

c) Diferença entre duas médias de tratamentos A de $\underline{n}$ tro de um mesmo nivel do tratamento B:

$$
\hat{\mathrm{Y}}_{i / k}=\left(\mathrm{a}_{1} \mathrm{~b}_{1}-\mathrm{a}_{0} \mathrm{~b}_{1}\right) \quad \hat{\mathrm{V}}\left(\hat{\mathrm{Y}}_{\mathrm{i} / \mathrm{k}}\right)=\frac{2\left[(\beta-1) \mathrm{E}_{c}+\mathrm{E}_{\mathrm{a}}\right]}{\mathrm{r} \beta}
$$

d) Diferença entre duas médias de tratamentos B dentro de um mesmo nível de tratamento A:

$$
\hat{Y}_{k / i}=\left(a_{1} b_{1}-a_{1} b_{0}\right) \quad \hat{v}\left(\hat{Y}_{k / i}\right)=\frac{2\left[(\alpha-1) E_{c}+E_{b}\right]}{r \alpha}
$$


Para ZIMMERMANN (1973), as comparações entre duas médias, para cada periodo, baseada no modelo

$$
y_{i j k}=m+\rho_{j}+a_{i}+\alpha_{i j}+b_{k}+\beta_{j k}+(a b)_{i k}+\varepsilon_{i j k}
$$

podem ser feitas de cinco diferentes maneiras, tendo as variancias de cada tipo de contraste a expressão:
a) $\hat{Y}_{i}=\bar{A}_{i}-\bar{A}_{i}$,
$\vec{V}\left(\hat{Y}_{i}\right)=\frac{2}{J K}[Q M \operatorname{Residuo}(a)]$
b) $\hat{\mathrm{Y}}_{\mathrm{k}}=\overline{\mathrm{B}}_{\mathrm{k}}-\overline{\mathrm{B}}_{\mathrm{k}^{\prime}}$
$\widehat{V}\left(\widehat{Y}_{k}\right)=\frac{2}{I J}[Q M \operatorname{Residuo}(b)]$
c) $\hat{Y}_{i / k}=\overline{A_{i} B_{k}}-\overline{A_{i}, B_{k}}$
$\hat{V}\left(\hat{Y}_{i / k}\right)=\frac{2}{J K}\left[\begin{array}{l}Q M \operatorname{Residuo}(a)+ \\ +(K-I) \text { QM Residuo }(c)\end{array}\right]$
d) $\widehat{Y}_{k / i}=\widehat{A}_{i B_{k}}-\overline{A_{i} B_{k}}$
$\widehat{V}\left(\hat{\mathrm{Y}}_{k / i}\right)=\frac{2}{I J}\left[\begin{array}{l}Q M \operatorname{Residuo}(b)+ \\ +(I-1) \text { QM Residuo (c) }\end{array}\right]$
e) $\hat{Y}_{i k}=\overline{A_{i} B_{k}}-\overline{A_{i}, B_{k}}$
$\widehat{V}\left(\hat{Y}_{i k}\right)=\left\{\begin{array}{l}I[Q M \operatorname{Residuo}(a)]+K[Q M \operatorname{Residuo~(b)~}]+ \\ +(I K-I-K) \text { QM Residuo (c) }\end{array}\right\}$ 
23.

\section{DESENVOLVIMENTO TEORICO}

Para o desenvolvimento teórico considerou-se um experimento em faixas com subparcelas subdivididas ten do-se I tratamentos $T$ e $K$ tratamentos $T^{\prime}$ dispostos nas par celas principais em faixas; L tratamentos $T *$ dispostos em cada subparcela resultante das combinações TT' e J blocos.

\section{1 - Mode 10 Matemätico}

$$
\begin{aligned}
& \text { Considerou-se o modelo linear: } \\
& y_{i k \ell j}=\mu+t_{i}+b+(t b)_{i j}+t_{k}^{\prime}+\left(t^{\prime} b\right)_{k j}+t t_{i k}^{\prime}+
\end{aligned}
$$

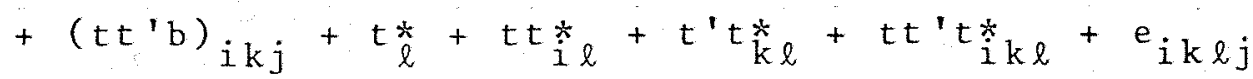

$$
\begin{aligned}
& \operatorname{com} i=1,2, \ldots, I \text {; } \\
& \mathrm{j}=1,2, \ldots, \mathrm{J} \text {; } \\
& \mathrm{k}=1,2, \ldots, \mathrm{k} \text {; } \\
& \ell=1,2, \ldots, \mathrm{L} \text {. }
\end{aligned}
$$


onde, $y_{i k \ell j}=$ valor observado da ikl-ésima subparcela no j-ésimo bloco;

$\mu=$ média geral;

$t_{i}=$ efeito do i-ésimo tratamento T;

$b_{j}=$ efeito aleatório do j-ésimo bloco, onde se supõe ${ }_{j} \sim N\left(0 ; \sigma_{b}^{2}\right)$;

$(t b)_{i j}=$ componente aleatório da interacão entre o i-ésimo tratamento $T$ e o j-ésimo bloco, onde se supõe $(t b)_{i j} \sim N\left(0 ; \sigma_{t b}^{2}\right)$;

$\mathrm{t}_{\mathrm{k}}^{\prime}=$ efeito do k-èsimo tratamento $\mathrm{T}^{\prime}$;

$\left(t^{\prime} b\right)_{k j}=$ componente aleatório da interação sentre o k-ésimo tratamento $T^{\prime}$ com o j-ésimo bloco onde se supõe $\left(t^{\prime} b\right)_{k j} \sim N\left(0 ; \sigma^{2} t^{\prime} b\right.$;

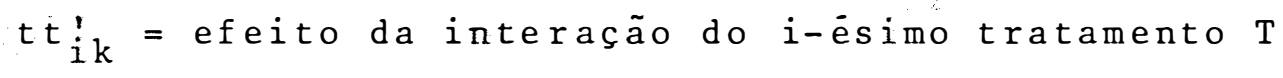
com o k-ésimo tratamento $T^{\prime}$;

$\left(t t^{\prime} b\right)_{i k j}=$ componente aleatório da interação entre o i-ésimo tratamento $T$ com o k-ésimo tratamen to $T^{\prime}$ no j-ésimo bloco, onde se supõe

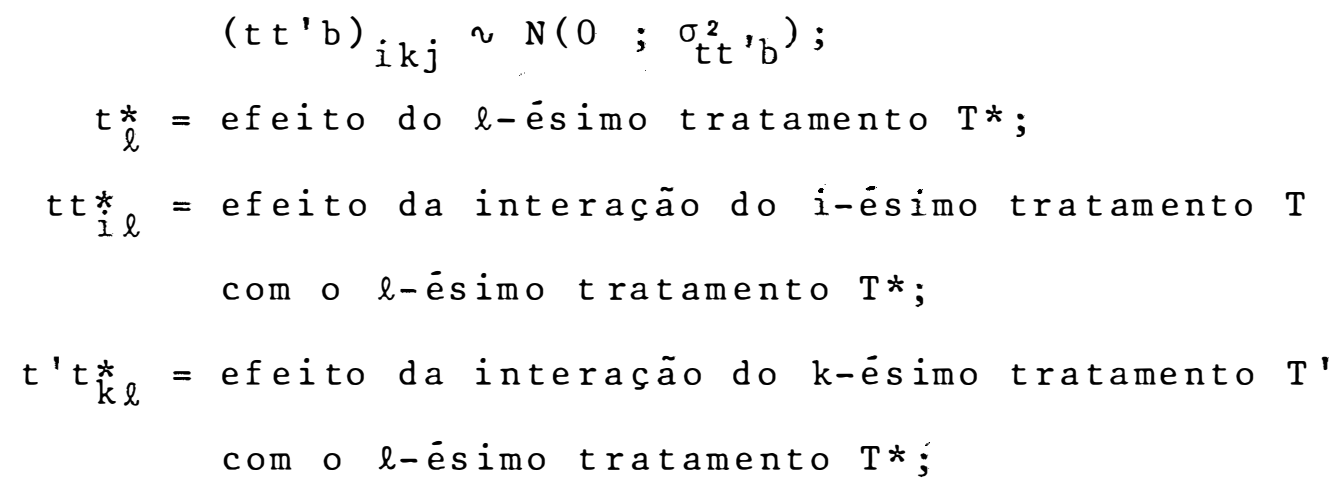


t t ${ }^{\prime}$ सkl $_{k \ell}=$ efeito da interação do i-ésimo tratamento $\mathrm{T}$ com o k-ésimo tratamento $T^{\prime} \operatorname{com} o l$-ësimo tra tamento $T^{*}$;

$e_{i k \ell j}=$ erro associado à iklj-ésima observação, onde se supõe $e_{i k \ell j} \sim N\left(0 ; \sigma^{2}\right)$.

A adoção da aleatoriedade para blocos foi fei ta com base em ANDERSON e BANCROFT (1952) e FEDERER (1955) que preconizam a alleatoriedade dos blocos, pois as inferén cias feitas a partir deles, valem para a população, e somente o consideram fixo quando as värias amostras são toma das de cada tratamento em cada bloco.

Resultou, assim, um modelo misto onde são con siderados aleatórios:

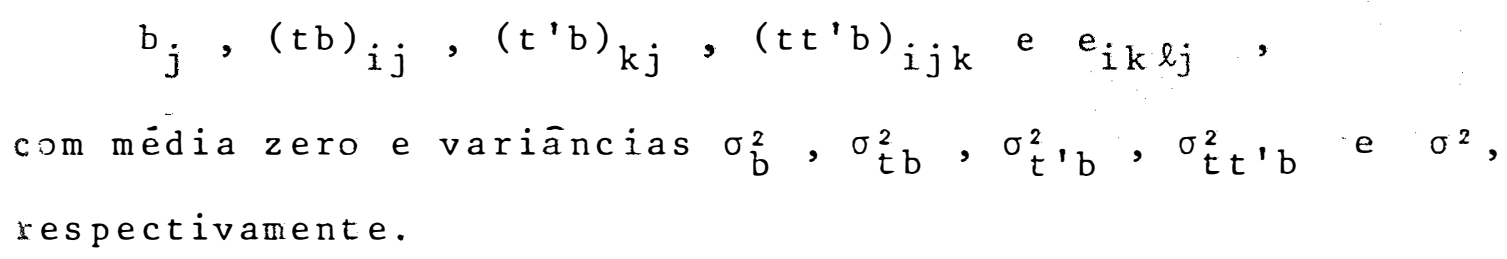

3.2 - Sistema de Equações Normais

3.2.1 - Obtenção do sistema de equações normais

o sistema de equações normais foi obtido atra Vés do método dos mínimos quadrados, resultando: 
a) Equação normal para média:

$$
\begin{aligned}
& \sum_{i j k \ell} y_{i k \ell j}=I J K L \bar{m}+J K L \sum_{i} \bar{E}_{i}+I K L \sum_{j} \sigma_{j}+K L \sum_{i j}(t b)_{i j}+ \\
& +I J L \sum_{k} E_{k}^{\prime}+I L \sum_{j k}\left(t \hat{T}^{\prime} b\right)_{k j}+ \\
& +J L \sum_{i k} t t_{i k}^{\prime}+L \sum_{i k j}\left(\widehat{t t^{\prime} b}\right)_{i k j}+
\end{aligned}
$$

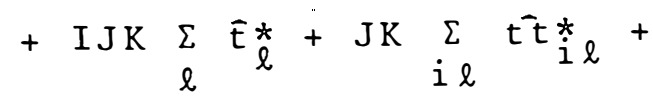

$$
\begin{aligned}
& +I J \sum_{k l} \widehat{t^{\top} t}{ }_{k l}^{*}+J \sum_{i k l} \widehat{t t^{\top} t}{ }_{i k l}^{*}
\end{aligned}
$$

b) equação normal para tratamento $T$ :

$$
\begin{aligned}
& \sum_{j k \ell} y_{i k \ell j}=J K L \bar{m}+J K L \bar{t}_{i}+K L \sum_{j} b_{j}+K L \sum_{j}(t b)_{i j}+ \\
& +J L \sum_{k} \bar{t}_{k}^{\prime}+L \sum_{j k}\left(\widehat{t^{\top} b}\right)_{j k}+J \sum_{k} \bar{t}_{i k}^{\prime}+ \\
& +L \sum_{j k}\left(t \widehat{t^{\top} b}\right)_{i k j}+J K \sum_{l} \bar{t}_{l}^{*}+
\end{aligned}
$$

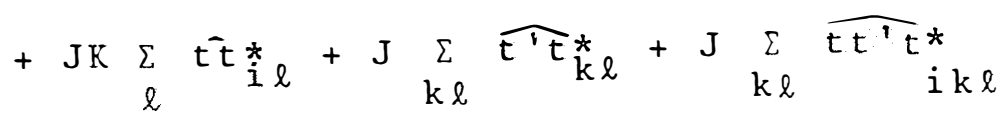

c) Equação normal para bloco

$$
\begin{aligned}
& \sum_{i k \ell} y_{i k \ell j}=I K L \bar{m}+K L \sum_{i} \bar{t}_{i}+I K L b_{j}+K L \sum_{i}(t b)_{i j}+ \\
& +L \sum_{i k} \bar{t} \bar{t}_{i k}+L \sum_{i k}\left(\widehat{t t^{\top} b}\right)_{i k j}+ \\
& +\operatorname{IK} \sum_{\ell} \bar{t}_{\ell}^{*}+K \sum_{i \ell} t_{i l}^{*}+I \sum_{k \ell} \widehat{t^{-1} t k_{k}^{*}}+ \\
& +\sum_{i k \ell} \widehat{t t^{\top} t} k_{i k \ell}+I L \sum_{k} E_{k}^{\prime}+I L \sum_{i}\left(\widehat{t^{\prime} b}\right)_{k j}
\end{aligned}
$$


d) Equação normal para a interação tratamento $T \times$ Bloco [Residuo(a)]:

$$
\begin{aligned}
& \sum_{k \ell} y_{i k \ell j}=K L \bar{m}+K L \bar{t}_{i}+K L b_{j}+K L(t b)_{i j}+L_{k} \bar{t}_{k}^{\prime}+ \\
& +L \sum_{k}\left(t^{\top} b\right)_{k j}+L \sum_{k} t^{\prime} i_{i k}^{\prime}+\sum_{k}\left(\widehat{t t^{\prime} b}\right)_{i j k}+
\end{aligned}
$$

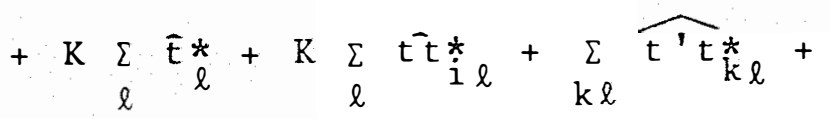

$$
\begin{aligned}
& +\sum_{k \ell} \widehat{t t}^{\prime}{ }_{i k \ell}^{*}
\end{aligned}
$$

e) Equação normal para tratamento $T^{\prime}$ :

$$
\begin{aligned}
& \sum_{i j \ell} y_{i k \ell j}=I J L \bar{m}+J L \sum_{i} \bar{t}_{i}+I L \sum_{j} b_{j}+L \sum_{i j}(t b)_{i j}+ \\
& +I J L \bar{t}_{k}^{\prime}+I L \sum_{j}\left(\hat{t}^{\top} b\right)_{k j}+J L \sum_{i} \bar{t}_{i k}^{\prime}+ \\
& +L \sum_{i j}\left(\widehat{t t^{\top} \bar{b}}\right)_{i k j}+I J \sum_{\ell} \overline{\hat{t}} \frac{i}{\hat{l}}+ \\
& +J \sum_{i \ell} t^{-} \hat{i}_{\ell}^{*}+I J \sum_{\ell} \widehat{t^{\top} t_{\hat{k} \ell}^{*}}+ \\
& +J \sum_{i \ell} \widehat{t t^{\prime} t_{i k l}^{*}} .
\end{aligned}
$$

f) Equação normal para a interação tratamento $T^{\prime} x$ Bloco [Residuo(b)]:

$$
\begin{aligned}
& \sum_{i \ell} y_{i k \ell j}=I L \bar{m}+L \sum_{i} \bar{t}_{i}+I L \bar{b}_{j}+L \sum_{i}(t b)_{i j}+I L \bar{t}_{k}^{\prime}+ \\
& +I L\left(t^{\prime} b\right)_{k j}+L \sum_{i} t^{\prime} i_{i k}^{\prime}+L \sum_{i}\left(\widehat{t t^{\prime} b}\right)_{i k j}+
\end{aligned}
$$

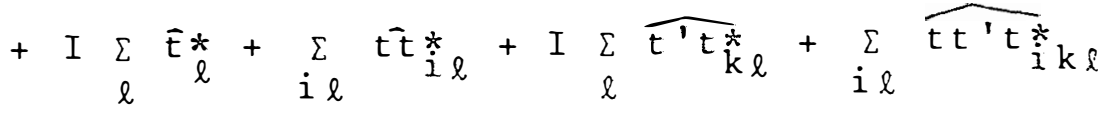


g) Equação normal para a interação $T \times T^{\prime}$ :

$$
\begin{aligned}
& \sum_{j \ell} y_{i k \ell j}=J L \hat{m}+J L \bar{t}_{i}+L \sum_{j} b_{j}+L \sum_{j}(t b)_{i j}+J L \bar{t}_{k}^{\prime}+ \\
& +L \sum_{j}\left(t^{\prime} b\right)_{k j}+J L t_{i k}+L \sum_{j}\left(\widehat{t t^{\prime} b}\right)_{i k j}+ \\
& +J \sum_{\ell} \bar{t}_{\ell}^{*}+J \sum_{\ell} t_{i}^{*} \underset{i l}{*}+J \sum_{\ell} \widehat{t t_{k}^{*}}+ \\
& +J \sum_{\ell} \widehat{t t^{\top} t * i k l}
\end{aligned}
$$

h) Equação normal para a interação $T \times T^{\prime} \times B$ [Resíduo(c)]:

$$
\begin{aligned}
& \sum_{\ell} y_{i k \ell j}=L \bar{m}+L \hat{t}_{i}+L b_{j}+L(t b)_{i j}+L \hat{t}_{k}^{\prime}+L\left(t^{\top} b\right)_{k j}+
\end{aligned}
$$

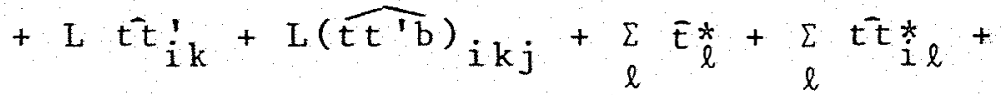

$$
\begin{aligned}
& +\sum_{l} \widehat{t t_{k l}^{*}}+\sum_{\ell} \widehat{t t}_{i k l}^{*}
\end{aligned}
$$

i) Equasão normal para tratamento $T *$ :

$$
\begin{aligned}
& \sum_{i j k} y_{i k \ell j}=I J K \bar{m}+J K \sum_{i} \hat{t}_{i}+I K \sum_{j} \sigma_{j}+K \sum_{i j}(t b)_{i j}+ \\
& +I J \sum_{k} \hat{t}_{k}^{\prime}+I \sum_{j k}\left(t^{\prime} b\right)_{k j}+J \sum_{i k} t^{\prime} i_{k}^{\prime}+ \\
& +\sum_{i k j}\left(\widehat{t t^{\top} b}\right)_{i k j}+I K J \hat{t}_{l}^{*}+J K \sum_{i} t_{i l}^{*}+ \\
& +I J \sum_{k} \widehat{t^{\top} t k l}+J \sum_{i k} \widehat{t^{\top} t}{ }_{i k l}^{*} \cdot
\end{aligned}
$$


j) Equação normal para a interação TxT*:

$$
\begin{aligned}
& \sum_{j k} y_{i k \ell j}=J K \bar{m}+J K \bar{t}_{i}+K \sum_{j}^{\sum} b_{j}+K \sum_{j}(t b)_{i j}+J \sum_{k} \bar{t}_{k}^{\prime}+ \\
& +\sum_{j k}\left(t^{i} b\right)_{k j}+J \sum_{k}^{\sum} t^{i} i_{i k}+\sum_{j k}\left(\widehat{t t^{\prime} b}\right)_{i k j}+
\end{aligned}
$$

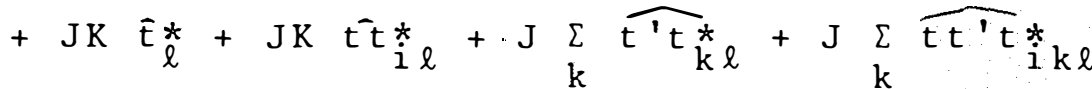

1) Equação normal para a interação $T^{\prime} x T^{*}$ :

$$
\begin{aligned}
& \sum_{i j} y_{i k \ell j}=I J \bar{m}+J \sum_{i} \bar{t}_{i}+I \sum_{j} \bar{b}_{j}+\sum_{i j}^{\sum}(t b)_{i j}+I J \hat{E}_{k}^{\prime}+ \\
& +I \sum_{j}\left(t^{\prime} b\right)_{k j}+J \sum_{i} t^{t} i_{i k}+\sum_{i j}\left(\widehat{t t^{\prime} b}\right)_{i k j}+ \\
& +I J \bar{t}_{\ell}^{*}+J \sum_{i} t_{i}^{*}{ }_{i l}^{*}+I J t^{\prime} t_{k \ell}^{*}+J \sum_{i} \widehat{t t}^{\top} t_{i k l}^{*}
\end{aligned}
$$

m) Equação normal para a interação TxT'xT*:

$$
\begin{aligned}
& \sum_{j} y_{i k \ell j}=J \hat{m}+J \bar{t}_{i}+\sum_{j} \bar{b}_{j}+\sum_{j}(t \hat{b})_{i j}+J \hat{E}_{k}^{\prime}+\sum_{j}(t \hat{b})_{k j}+
\end{aligned}
$$

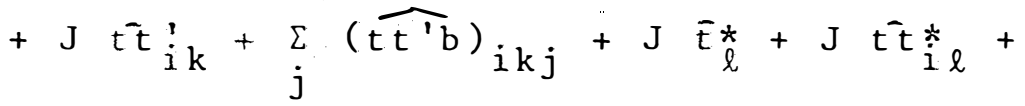

$$
\begin{aligned}
& +J \widehat{t t_{k \ell}^{k}}+J \widehat{t t^{\prime}} \widehat{i}_{i k \ell}^{k}
\end{aligned}
$$

Consideraram-se as seguintes restrições: 


$$
\begin{aligned}
& \sum_{1} \bar{E}_{i}=0 \\
& \sum_{\mathrm{k}} \overline{\mathrm{E}}_{\mathrm{k}}=0 \\
& \sum_{\ell} \bar{E} *=0 \\
& \sum_{j} \sigma_{j}=0 \\
& \sum_{i} t^{-t} i k_{k}=0 \\
& \sum_{k} t^{-} t_{i k}^{\prime}=0 \\
& \sum_{k} t{ }^{\top} t \frac{k}{k l}=0 \\
& \sum_{i} t_{i l}{ }_{i}^{*}=0 \\
& \sum_{\ell} t^{-}{ }_{i \ell}^{*}=0
\end{aligned}
$$

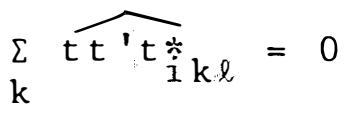

$$
\begin{aligned}
& \sum_{\ell} t^{\top} t \underset{k \ell}{*}=0 \\
& \sum_{i} \widehat{t t^{\prime} t k l}=0 \\
& \sum_{j} t b_{i j}=0 \\
& \sum_{\ell} \widehat{t t^{\prime} t *}{ }_{i k l}^{*}=0 \\
& \sum_{i}(t b)_{i . j}=0 \\
& \sum_{i} \widehat{t}^{\prime} b_{i k j}=0 \\
& \sum_{k}{\widehat{t} \cdot b_{k j}}_{k j}=0 \\
& \sum_{j}{\widehat{t} \cdot b_{k j}}_{k}=0 \\
& \sum_{k}{\widehat{t t^{\prime} b}}_{i j k}=0 \\
& \sum_{j} t \widehat{t^{\prime} b_{i k j}}=0
\end{aligned}
$$

e desse modo, o sistema de equaçóes normais ficou:

$$
\begin{aligned}
& \operatorname{JKL} \overline{\mathrm{m}}+\operatorname{JKL} \hat{\mathrm{t}}_{i}=\mathrm{T}_{i} \\
& \operatorname{IKL} \overline{\mathrm{m}}+\operatorname{IKL} \bar{b}_{j}=B_{j}
\end{aligned}
$$

$$
K L \bar{m}+K L \bar{t}_{i}+K L \bar{b}_{j}+K L(t b)_{i j}=y_{i} \ldots j
$$


31.

$$
\text { IJLîm IJL } t_{\hat{k}}^{\prime}=T_{k}^{\prime}
$$

$$
I L \bar{m}+I L \bar{t}_{k}^{\prime}+I L B_{j}+I L(\widehat{t ' b})_{k j}=y \cdot k \cdot j
$$

$$
J L \bar{m}+J L \bar{t}_{i}+J L \bar{t}_{k}^{\prime}+J L \bar{t}_{i k}^{\prime}=T_{T k}^{\prime}
$$

$L \bar{m}+L \bar{t}_{i}+L \hat{b}_{j}+L(t b)_{i j}+L \hat{E}_{k}^{\prime}+L(\widehat{t b})_{k j}+L t_{i k}^{\prime}+L(\widehat{t E} b)_{1 k j}=y_{i k \cdot j}$

$$
I J K^{-}+I J K E_{\ell}^{*}=T_{\ell}^{*}
$$

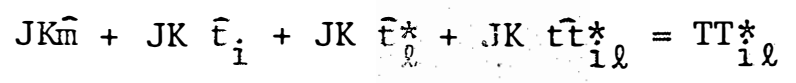

$$
I J \bar{m}+I J \hat{t}_{k}^{\prime}+I J t_{\ell}^{*}+I \cdot T \widehat{t}_{t_{k \ell}^{*}}^{*}=T^{\prime} T_{k \ell}^{*}
$$

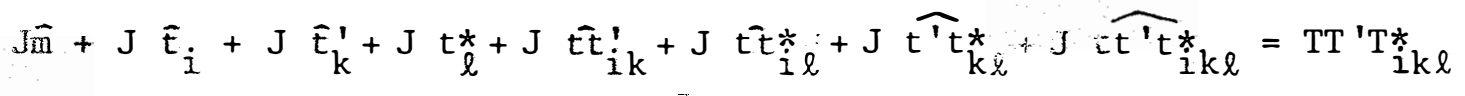

$$
\text { onde, } G=\text { total geral; }
$$$$
\mathrm{T}_{i}=\text { total do i-ésimo tratamento } \mathrm{T} \text {; }
$$$$
B_{j}=\text { total do j-ésimo bloco; }
$$$$
y_{i . j}=\text { total das parcelas que recebereno i-ésimo tra- }
$$
tamento T no j-ésimo bloco;

$\mathrm{T}_{\mathrm{k}}^{\prime}=$ total do k-ésimo tratamento $\mathrm{T}^{\prime}$

y.k.j = total das parcelas que receberamo k-ésimo tratamento T' no j-ésimo bloco;

$$
\begin{aligned}
\mathrm{TT}_{\text {ik }}^{\prime}= & \text { total das subparcelas relativas ao i-ësimo tra- } \\
& \text { tamento } \mathrm{T} \text { com o k-ësimo tratamento T'. }
\end{aligned}
$$




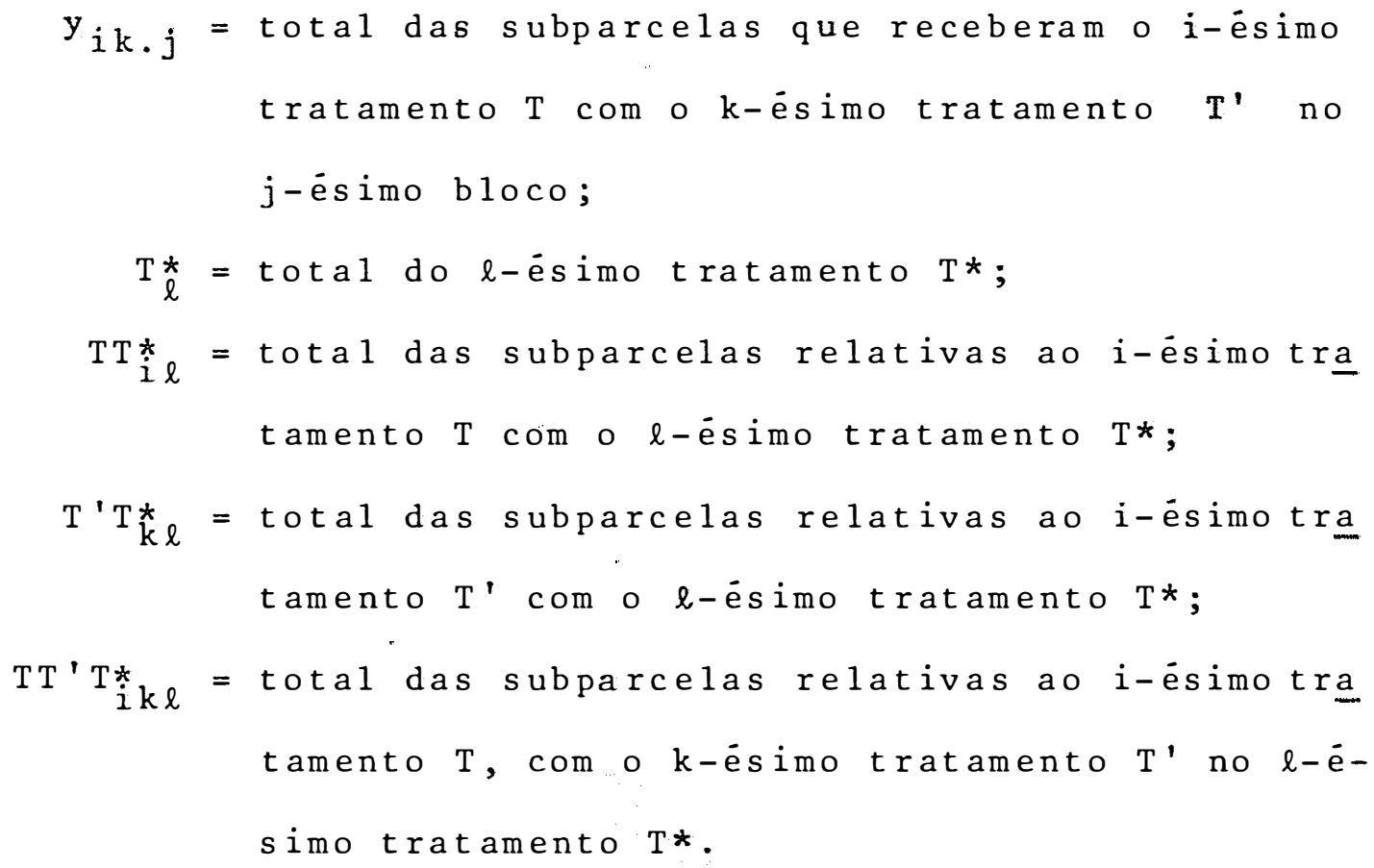

a) Para média gera 1:

$$
\overline{\mathrm{m}}=\frac{\mathrm{G}}{\mathrm{IJKL}} \quad \text {; }
$$

b) Para o i-ésimo tratamento T:

$$
\overline{\mathrm{t}}_{i}=\frac{\mathrm{T}_{i}}{J K L}-\overline{\mathrm{m}} ;
$$


c) Para o j-ésimo bloco:

$$
\sigma_{j}=\frac{B_{j}}{I K L}-\bar{m}
$$

d) Para o i-ésimo tratamento $T$ como j-ésimo bloco:

$$
(t b)_{i j}=\frac{y_{i \cdot j_{j}}}{K L}-\bar{t}_{i}-b_{j}-\bar{m} ;
$$

e) Para o k-ésimo tratamento $T^{\prime}$ :

$$
\hat{E}_{k}^{\prime}=\frac{T_{k}^{\prime}}{I J L}-\hat{m} ;
$$

f) Para o k-ésimo tratamento $T^{\prime}$ como j-ésimo bloco:

$$
(t \widehat{b})_{k j}=\frac{y_{\cdot k \cdot j}}{I L}-\hat{t}_{k}^{\prime}-b_{j}-\hat{m} ;
$$

g) Para a interação do i-ésimo tratamento $T$ com o k-èsimo tratamento $\mathrm{T}^{\prime}$ : $t \bar{t}_{i k}=\frac{T T_{i k}^{\prime}}{J L}-\bar{t}_{i}-E_{k}^{\prime}-\hat{m} ;$

h) Para a interação do i-ésimo tratamento $T$ com o k-ésimo tratamento $\mathrm{T}^{\prime}$ no j-ésimo bloco:

$$
\begin{aligned}
\left(\widehat{t t^{\top} b}\right)_{i k j}=\frac{y_{i k} j}{L}-t_{i k}^{\prime}-\left(\widehat{t} \hat{T}_{k j}-\bar{t}_{k}^{\prime}-(\hat{t b})_{i j}-\right. \\
-\bar{b}_{j}-\hat{t}_{i}-\bar{m}^{\prime}
\end{aligned}
$$


i) Para o $\ell$-ésimo tratamento $T *$ :

$$
\hat{\mathrm{E}}_{\ell}^{*}=\frac{\mathrm{T}_{\ell}^{*}}{I \mathrm{JK}}-\hat{\mathrm{m}}
$$

j) Para a interação do i-ésimo tratamento $T$ com o $\ell$-ésimo tratamento T*:

$t_{i \ell}^{*}=\frac{T_{i}^{*} \ell}{J K}-\bar{t}_{\ell}^{*}-\bar{t}_{i}-\bar{m} ;$

1) Para a interação do k-ësimo tratamento T' com o $\ell$-ésimo tratamento T*:

${ }^{T} T_{k l}^{*}=\frac{T^{\prime} T_{k}^{*}}{I J}-\bar{t}_{k}^{\prime}-\bar{t}_{l}^{*}-\bar{m} ;$

m) Para a interação do i-ésimo tratamento $\mathrm{T}$ com o k-ésimo tratamento $T^{\prime}$ no $\ell$-ésimo tratamento $T *$ :

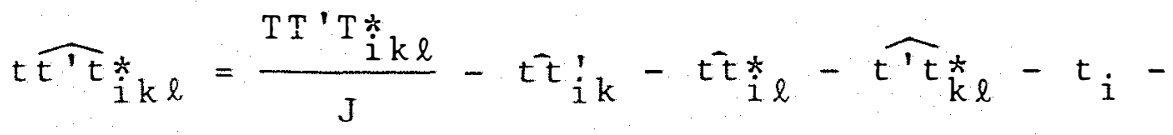

$$
\begin{aligned}
& -E_{k}^{\prime}-E_{l}^{k}-\bar{m} \text {. }
\end{aligned}
$$




\section{3 - Anālise de Variāncia}

\subsection{1 - Obtenção das somas de quadrados}

A soma de quadrados dos parāmetros foi obtida, segundo nogueira (1962), Pimentel Gomes (1966), dentre outros, atravēs da soma dos produtos das soluções pelos segundos membros das equações normais correspondentes, result ando:

a) $\mathrm{C}=\overline{\mathrm{m}} \mathrm{G}=\frac{\mathrm{G}^{2}}{\mathrm{IJKL}}$;

b) SQ Tratamento $\mathrm{T}=\mathrm{SQT}=\sum_{i} \hat{\mathrm{t}}_{\mathrm{i}} \mathrm{T}_{i}=\frac{1}{\mathrm{JKL}} \sum_{i} \mathrm{~T}_{i}^{2}-\mathrm{C}$;

c) $\mathrm{SQ} B$ loco $=\mathrm{SQB}=\sum_{j} \mathrm{~B}_{j} \mathrm{~B}_{j}=\frac{1}{I K L} \sum_{j} B_{j}^{2}-C$

d) $\operatorname{SQResiduo(a)}=\operatorname{SQR}(a)=\sum_{i j}(\widehat{t b})_{i j} y_{i . . j}=\frac{1}{K L} \sum_{i j} y_{i \ldots j}^{2}$

$$
-\mathrm{C}-\mathrm{SQT}-\mathrm{SQB} \text {; }
$$

e) $\mathrm{SQ}$ Tratamento $\mathrm{T}^{\prime}=\mathrm{SQT}^{\prime}=\sum_{\mathrm{k}} \overline{\mathrm{E}}_{\mathrm{k}}^{\prime} \mathrm{T}_{\mathrm{k}}^{\prime}=\frac{1}{\mathrm{IJL}} \sum_{\mathrm{k}}\left(\mathrm{T}_{\mathrm{k}}^{\prime}\right)^{2}-\mathrm{C}$;

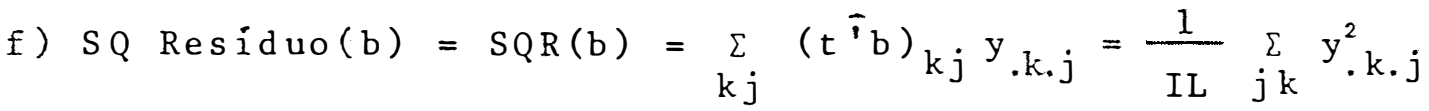

$$
-\mathrm{C}-\mathrm{SQT} \mathrm{T}^{\prime}-\mathrm{SQB} \text {; }
$$


g) SQ Interação $\operatorname{TxT}^{\prime}=\operatorname{SQTxT}^{\prime}=\sum_{i k} t_{i k}^{\prime} \mathrm{TT}_{i \mathrm{k}}^{\prime}=$ $=\frac{1}{J L} \sum_{i k}\left(T_{i k}^{\prime}\right)^{2}-C-S Q T-S Q T^{\prime}$

h) $S Q \operatorname{Residuo(c)}=S Q R(c)=\sum_{i k j}(t \widehat{t ' b})_{i k j} Y_{i k j}=$

$$
\begin{gathered}
=\frac{1}{L} \sum_{i j k} \dot{y}_{i k \cdot j}^{2}-C-S Q T-S Q B- \\
-S Q T^{\prime}-S Q T \times T^{\prime}-S Q R(a)-
\end{gathered}
$$$$
\text { - SQR(b) ; }
$$

i) $\mathrm{SQ}$ Tratamento $\mathrm{T}^{*}=\mathrm{SQT}^{*}=\sum_{l} \overline{\mathrm{E}}_{\ell}^{*} \mathrm{~T}_{\ell}^{*}=\frac{1}{\mathrm{IJK}} \sum_{l}\left(\mathrm{~T}_{\ell}^{*}\right)^{2}-\mathrm{C}$;

j) SQ Interação $\operatorname{TxT} * \operatorname{QTT} \times T^{*}=\sum_{i \ell} \mathrm{t}^{\top}{ }_{i \ell}^{*} \mathrm{TT}_{i \ell}^{*}=$

$$
=\frac{1}{\mathrm{JK}} \sum_{i \ell}\left(\mathrm{TT}_{\mathrm{i} \ell}^{*}\right)^{2}-\mathrm{C}-\mathrm{SQT}-
$$

$$
-\mathrm{SQT}^{*} \text {; }
$$

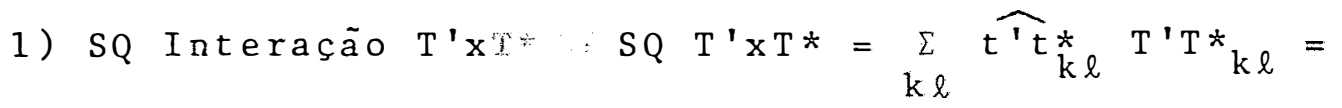

$$
\begin{aligned}
& =\frac{1}{\mathrm{IJ}} \sum_{\mathrm{k} \ell}\left(\mathrm{T}^{\prime} \mathrm{T}^{*}\right) \underset{\mathrm{k} \ell}{2}-\mathrm{C}- \\
& -\mathrm{SQT}^{\prime}-\mathrm{SQT} *
\end{aligned}
$$




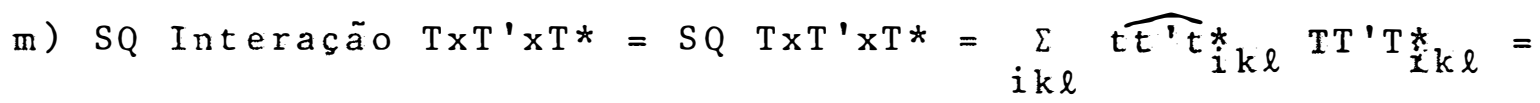

$$
\begin{aligned}
& =\frac{1}{J} \sum_{i k \ell}\left(\mathrm{TT}^{\prime} \mathrm{T}^{*}\right) \underset{i k \ell}{2}-\mathrm{C}-\mathrm{SQT}- \\
& -\mathrm{SQT}^{\prime}-\mathrm{SQT} *-\mathrm{SQT} \times \mathrm{T}^{\prime}-\mathrm{SQT \times T} \text { * }
\end{aligned}
$$

$-\mathrm{SQT}^{\prime} \mathrm{xT} *$

n) $\operatorname{SQTotal}=\sum_{i k \ell j} y_{i k \ell j}^{2}-C$;

o) $S Q \operatorname{Residuo~(d)=SQR(d)=SQTotal~-~SQT~-~SQB~-~SQT'~-~}$

$-\mathrm{SQT} *-\operatorname{SQT} \times T^{\prime}-\operatorname{SQT} \times \mathrm{T}^{*}-$

- SQT'xT* - SQTXT'xT* - SQR(a) -

- $\operatorname{SQR}(b)-\operatorname{SQR}(\mathrm{c})$. 
3.3.2 - Esperança matemática dos quadrados médios

Com base nas somas de quadrados obtidas em (3.3.1), no modelo 1 inear proposto em $(3.1)$ e através dos procedimentos descritos em PIMENTEL GOMES (1966), as espe ranças dos quadrados médios resultaram:

a) $E(\operatorname{correção~})=E(C)=E\left[\frac{\left(\sum \sum_{i k \ell} y_{i k \ell j}\right)^{2}}{I J K L}\right]$

Dado que :

$$
\begin{aligned}
y_{i k \ell j}=\mu & +t_{i}+b_{j}+(t b)_{i j}+t_{k}^{\prime}+\left(t b_{k j}+t t_{i k}^{\prime}+\right. \\
& +\left(t t^{\prime} b\right)_{i k j}+t \frac{k}{\ell}+t t_{i \ell}^{*}+t^{\prime} t t_{k \ell}^{*}+t t^{\prime} t_{i k \ell}^{*}+ \\
& +e_{i k \ell j},
\end{aligned}
$$

e pelo fato de que no resultado final das diversas E(SQ)

$\mu$ não aparece, pode-se suprimi-la em todos os cálculos, fa cilitando assim as deduções:

Assim,

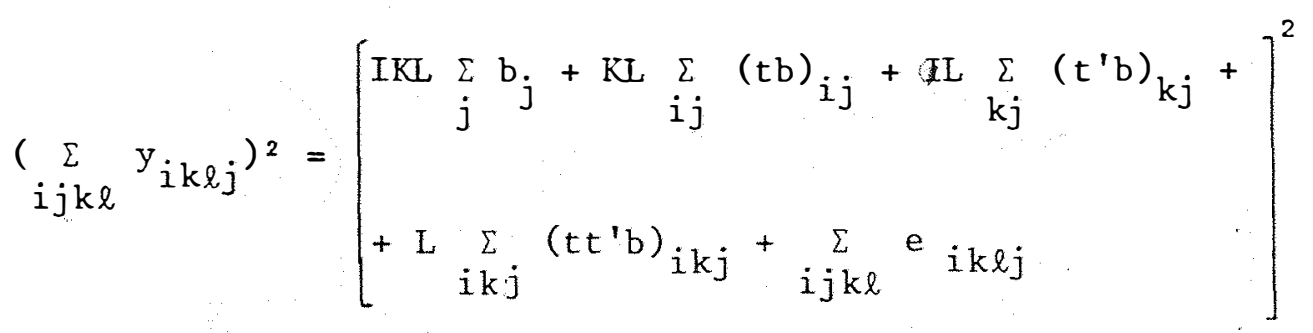




$$
\begin{aligned}
E\left[\left(\sum_{i j k \ell} y_{i k \ell j}\right)^{2}\right]=I^{2} J^{2} L^{2} \sigma_{b}^{2} & +I J K^{2} L^{2} \sigma_{t b}^{2}+I^{2} J K L^{2} \sigma_{t}^{2} b+ \\
& +I J K L^{2} \sigma_{t t^{\prime} b}^{2}+I J K L \sigma^{2}
\end{aligned}
$$

e

$$
\begin{aligned}
& E(C)=\frac{1}{I J K L}\left[\begin{array}{l}
I^{2} J K^{2} L^{2} \sigma_{b}^{2}+I J K^{2} L^{2} \sigma_{t b}^{2}+I^{2} J K L L^{2} \sigma_{t}^{2}{ }^{\prime} b+ \\
+I J K L^{2} \sigma_{t t^{\prime} b}^{2}+I J K L \sigma^{2}
\end{array}\right] \\
& \therefore E(C)=I K L \sigma_{b}^{2}+K L \sigma_{t b}^{2}+I L \sigma_{t}^{2} b+L \sigma_{t t}^{2}{ }^{\prime} b+\sigma^{2}
\end{aligned}
$$

b) Partindo-se de $\mathrm{QMT}=\frac{\mathrm{SQT}}{\mathrm{I}-1}$ tem-se:

$$
\mathrm{E}(\mathrm{QMT})=\frac{1}{\mathrm{I}-1} \mathrm{E}(\mathrm{SQT})
$$

onde

$$
\mathrm{SQT}=\frac{1}{\mathrm{JKL}} \sum_{\mathrm{i}} \mathrm{T}_{\mathrm{i}}^{2}-\mathrm{C}
$$

e

$$
\begin{aligned}
T_{i}=J K L t_{i} & +K L \sum_{j} b_{j}+K L \sum_{j}(t b)_{i j}+L \sum_{j k}\left(t^{\prime} b\right)_{k j}+ \\
& +L \sum_{j k}\left(t t^{\prime} b\right)_{i k j}+\sum_{i j k l} e_{i k j l}
\end{aligned}
$$

então,

$$
\frac{I}{J K L} E\left(\sum_{i} T_{i}^{2}\right)=J K L \underset{i}{\sum_{i}} t_{i}^{2}+I K L \sigma_{b}^{2}+I K L \sigma_{t b}^{2}+I L \sigma_{t}^{2}{ }^{\prime} b+I L \sigma_{t t^{\prime} b}^{2}+I \sigma^{2} .
$$




\section{Subtraindo-se a E(C) vem:}

$$
\begin{aligned}
\mathrm{E}(\mathrm{SQT})=\mathrm{JKL} \sum_{\mathrm{i}} \mathrm{t}_{\mathrm{i}}^{2} & +\mathrm{KL}(\mathrm{I}-1) \sigma_{\mathrm{tb}}^{2}+\mathrm{L}(\mathrm{I}-1) \sigma_{\mathrm{t} t}^{2} \mathrm{~b}+ \\
& +(\mathrm{I}-1) \sigma^{2}
\end{aligned}
$$

dividindo-se por (I-1)

$$
E(Q M T)=\frac{J K L}{(I-I)} \sum_{i} t_{i}^{2}+K L \sigma_{t b}^{2}+L \sigma_{t}^{2} t_{b}^{\prime}+\sigma^{2}
$$

c) Analogamente ao caso anterior $Q M B=\frac{S Q B}{J-1}$, assim:

$$
\mathrm{E}(\mathrm{QMB})=\frac{1}{(\mathrm{~J}-1)} \mathrm{E}(\mathrm{SQB}) \text {, }
$$

ande:

$$
S Q B=\frac{1}{I K L} \sum_{j} B_{j}^{2}-C
$$

e

$$
\begin{aligned}
B_{j}=I K L b_{j} & +K L \sum_{i}(t b)_{i j}+I L \sum_{k}\left(t^{\prime} b\right)_{k j}+ \\
& +\sum_{i k}\left(t t^{\prime} b\right)_{i k j}+\sum_{i k l} e_{i k l j}
\end{aligned}
$$

então:

$$
\begin{aligned}
\frac{1}{I K L} E\left(\sum_{j} B_{j}^{2}\right)=I J K L \sigma_{b}^{2} & +J K L \sigma_{t b}^{2}+I J L \sigma_{t}^{2} b+ \\
& +J L \sigma_{t t^{\prime} b}^{2}+J \sigma^{2},
\end{aligned}
$$

subtraindo-se a $\mathrm{E}(\mathrm{C})$ vem: 


$$
\begin{aligned}
E(S Q B)=I K L(J-1) \sigma_{b}^{2} & +K L(J-1) \sigma_{t b}^{2}+I L(J-1) \sigma_{t}^{2} \cdot b+ \\
& +L(J-1) \sigma_{t t^{\prime} b}^{2}+(J-1) \sigma^{2},
\end{aligned}
$$

dividindo-se por $(J-1)$,

$$
E(Q M B)=I K L \sigma_{b}^{2}+K L \sigma_{t b}^{2}+I L \sigma_{t}^{2}{ }^{\prime} b+L \sigma_{t t^{\prime} b}^{2}+\sigma^{2} .
$$

d) Partindo-se de $\operatorname{QMR}(\mathrm{a})=\frac{\mathrm{SQR}(\mathrm{a})}{(\mathrm{I}-1)(\mathrm{J}-1)}$, tem-se:

$$
\operatorname{E}[\operatorname{QMR}(a)]=\frac{1}{(I-1)(J-1)} \cdot \operatorname{E}[\operatorname{SQR}(a)]
$$

onde:

$$
\operatorname{SQR}(a)=\frac{1}{K L} \sum_{i j} y_{i . . j}^{2}-C-S Q T-S Q B
$$

e

$$
\begin{aligned}
y_{i \ldots j}=K L t_{i} & +K L b_{j}+K L(t b)_{i j}+L \sum_{k}\left(t^{\prime} b\right)_{k j}+ \\
& +L\left(t t^{\prime} b\right)_{i k j}+\sum_{k \ell} e_{i k \ell j},
\end{aligned}
$$

então:

$$
\begin{aligned}
\frac{1}{K L} E\left(\sum_{i j} y_{i . . j}^{2}\right)=J K L \sum_{i} t_{i}^{2} & +I J K L \sigma_{b}^{2}+I J K L \sigma_{t b}^{2}+ \\
& +I J L \sigma_{t}^{2} b+I J L \sigma_{t t^{\prime} b}^{2}+I J \sigma^{2}
\end{aligned}
$$

Subtraindo-se a E(C), E(SQT) e E(SQB), vem: 


$$
\begin{aligned}
E[S Q R(a)]=K L(I-1)(J-1) \sigma_{t b}^{2} & +L(I-1)(J-1) \sigma_{t}^{2} t^{\prime} b+ \\
& +(I-1)(J-1) \sigma^{2}
\end{aligned}
$$

$$
\begin{aligned}
& \text { Dividindo-se por }(\mathrm{I}-1)(\mathrm{J}-1): \\
& \mathrm{E}[\mathrm{QMR}(\mathrm{a})]=\mathrm{KL} \sigma_{t b}^{2}+\mathrm{L}_{t \mathrm{t}}^{2} \mathrm{~b}+\sigma^{2}
\end{aligned}
$$

e) Analogamente ao caso anterior: QMT' $=\frac{\mathrm{SQT}^{\prime}}{\mathrm{K}-1}$, então:

$$
E\left(Q M^{\prime}\right)=\frac{1}{(K-1)} E\left(S Q T^{\prime}\right) \text {, }
$$

onde:

$$
S Q T^{\prime}=\frac{1}{I J L} \sum_{k} T_{k}^{2}-C
$$

e

$$
\begin{aligned}
T_{k}^{\prime}=I L \sum_{j} b_{j} & +I J L t_{k}^{\prime}+L_{i j}(t b)_{i j}+I L \sum_{j}\left(t^{\prime} b\right)_{k j}+ \\
& +\sum_{i j}\left(t t^{\prime} b\right)_{i k j}+\sum_{i j l} e_{i k l j},
\end{aligned}
$$

então:

$$
\begin{aligned}
\frac{1}{\text { IJL }} E_{k}\left(\Sigma_{k} T_{k}^{2}\right)=I K L \sigma_{b}^{2} & +K L \sigma_{t b}^{2}+I J L_{k}^{\sum} t_{k}^{2}+I K L \sigma_{t}^{2} b+ \\
& +K L \sigma_{t t^{\prime} b}^{2}+K \sigma^{2},
\end{aligned}
$$

subtraindo-se a $E(C)$, vem:

$$
\begin{aligned}
E\left(S Q T^{\prime}\right)=I J L \sum_{k} t_{k}^{2} & +I L(K-1) \sigma_{t^{\prime} b}^{2}+L(K-1) \sigma_{t t^{\prime} b}^{2}+ \\
& +(K-1) \sigma^{2}
\end{aligned}
$$


dividindo-se por $(\mathrm{K}-1)$ :

$$
\mathrm{E}\left(\mathrm{QMT} \mathrm{T}^{\prime}\right)=\frac{\mathrm{IJL}}{\mathrm{K}-1 \mathrm{k}} \sum_{\mathrm{k}} \mathrm{t}_{\mathrm{k}}^{2}+\mathrm{IL} \sigma_{\mathrm{t}}^{2} \mathrm{~b}+\mathrm{L}_{\mathrm{t} \mathrm{t}^{\prime} \mathrm{b}}+\sigma^{2}
$$

f) Partindo-se de QMR(b) $=\frac{\mathrm{SQR}(\mathrm{b})}{(\mathrm{J}-\mathrm{l})(\mathrm{K}-1)}$, tem-se:

$$
\mathrm{E}[\mathrm{QMR}(\mathrm{b})]=\frac{1}{(\mathrm{~J}-\mathrm{I})(\mathrm{K}-1)} \mathrm{E}[\mathrm{SQR}(\mathrm{b})]
$$

onde:

$$
\mathrm{SQR}(\mathrm{b})=\frac{1}{\mathrm{IL} \quad \mathrm{kj}} \mathrm{y}^{2} \cdot \mathrm{k} \cdot \mathrm{j}^{-} \mathrm{C}-\mathrm{SQT}^{\prime}-\mathrm{SQB}
$$

e,

$$
\begin{aligned}
y_{\cdot k \cdot j}=I L t_{k}^{\prime} & +I L b_{j}+L \sum_{i}(t b)_{i j}+I L(t ' b)_{k j}+ \\
& +L \sum_{i}\left(t t^{\prime} b\right)_{i k j}+\sum_{i j} e_{i k \ell j}
\end{aligned}
$$

então:

$$
\begin{aligned}
& \frac{1}{I L} E\left(\sum_{k j}^{\sum_{j k}} y_{. j}^{2}\right)=I J L \sum_{k} t_{k}^{2}+I J K L \sigma_{b}^{2}+J K L \sigma_{t b}^{2}+ \\
& +\mathrm{IJKL} \sigma_{\mathrm{t} \cdot \mathrm{b}}^{2}+\mathrm{JKL} \sigma_{\mathrm{t} \mathrm{t}^{\prime} \mathrm{b}}^{2}+\mathrm{JK} \sigma^{2} \\
& \text { Subtraindo-se a } E(C), E\left(S Q T^{\prime}\right) \text { e } E(S Q B) \text {, vem: } \\
& E[S Q R(b)]=\operatorname{IL}(J-1)(K-1) \quad \sigma_{t}^{2}{ }^{\prime} b+ \\
& +\mathrm{L}(\mathrm{J}-1)(\mathrm{K}-1) \sigma_{\mathrm{t} \mathrm{t}^{\prime} \mathrm{b}}^{2}+ \\
& +(\mathrm{J}-1)(\mathrm{K}-1) \sigma^{2} .
\end{aligned}
$$


44.

$$
\text { Dividindo-se por }(\mathrm{J}-1)(\mathrm{K}-1) \text { : }
$$

$$
E[Q M R(b)]=I L \sigma_{t}^{2}{ }^{\prime} b+L \sigma_{t t^{\prime} b}^{2}+\sigma^{2}
$$

g) Analogamente ao caso anterior: QMTxT' $^{\prime}=\frac{\text { SQTxT' }^{\prime}}{(\mathrm{I}-1)(\mathrm{K}-1)}$, en tão:

$$
E\left(Q M T \times T^{\prime}\right)=\frac{1}{(I-1)(K-1)} E\left(\operatorname{SQT} \times T^{\prime}\right)
$$

onde:

$$
\mathrm{SQTxT}^{\prime}=\frac{1}{\mathrm{JL}} \sum_{i k}\left(\mathrm{TT}_{i k}^{\prime}\right)^{2}-\mathrm{C}-\mathrm{SQT}-\mathrm{SQT} \mathrm{T}^{\prime}
$$

e

$$
\begin{aligned}
\mathrm{TT}_{i k}^{\prime}=\mathrm{JL} \mathrm{t}_{i} & +\mathrm{L} \sum_{j} \mathrm{~b}_{j}+\mathrm{L} \sum_{j}(\mathrm{tb})_{i j}+\mathrm{JL} \mathrm{t}_{k}^{\prime}+ \\
& +\mathrm{L} \sum_{j}\left(t^{\prime} \mathrm{b}\right)_{k j}+J L t_{i k}^{\prime}+ \\
& +\mathrm{L} \sum_{j}\left(t t^{\prime} b\right)_{i k j}+\sum_{j \ell} e_{i k \ell j},
\end{aligned}
$$

ent ào:

$$
\begin{aligned}
& \frac{1}{J L} E\left[\sum_{i k}\left(\mathrm{TT}_{i k}^{\prime}\right)^{2}\right]=\mathrm{JKL} \sum_{i} t_{i}^{2}+I K L \sigma_{b}^{2}+I K L \sigma_{t b}^{2}+ \\
& +\operatorname{IJL} \sum_{k} t_{k}^{2}+I K L \sigma_{t}^{2} b^{+}+ \\
& +J L \sum_{i k}\left(t t^{\prime}\right) \underset{i k}{2}+\operatorname{IKL} \sigma_{t t^{\prime} b}^{2}+ \\
& +\operatorname{IK} \sigma^{2}
\end{aligned}
$$




$$
\begin{aligned}
& \text { Subtraindo-se a } E(C), E(S Q T) \text { e } E\left(S Q T^{\prime}\right) \text { vem: } \\
& E\left(S Q T \times T^{\prime}\right)=J_{L} \sum_{i k}\left(t t_{i k}^{\prime}\right)^{2}+L(I-1)(K-1) \sigma_{t t^{\prime} b}^{2}+ \\
& +(I-1)(K-1) \sigma^{2},
\end{aligned}
$$

dividindo-se por $(\mathrm{I}-1)(\mathrm{K}-\mathrm{I})$ :

$$
\left.\mathrm{E}(\mathrm{QMT \times T})^{\prime}\right)=\frac{\mathrm{JL}}{(\mathrm{I}-1)(\mathrm{K}-1) \sum_{i k}}\left(\mathrm{t} \mathrm{i}_{i k}^{\prime}\right)^{2}+\mathrm{L}_{\mathrm{t} \mathrm{t}^{\prime} \mathrm{b}}+\sigma^{2}
$$

h) Partindo-se de $Q M R(c)=\frac{S Q R(c)}{(I-1)(K-1)(J-1)}$, tem-se:

$$
\left.\operatorname{E}[\mathrm{QMR}(\mathrm{c})]=\frac{1}{(\mathrm{I}-1)(\mathrm{K}-\mathrm{I})(\mathrm{J}-1)} \operatorname{E[SQR}(\mathrm{c})\right]
$$

onde:

$$
\begin{aligned}
\mathrm{SQR}(\mathrm{c})=\frac{1}{\mathrm{~L}} \mathrm{ijk}_{\mathrm{jk}, \mathrm{j}} \mathrm{y}_{\mathrm{ik}}-\mathrm{C}-\mathrm{SQT}-\mathrm{SQT}-\mathrm{SQB}- \\
-\mathrm{SQT \times T}-\mathrm{SQR}(\mathrm{a})-\mathrm{SQR}(\mathrm{b})
\end{aligned}
$$

e

$$
\begin{aligned}
y_{i k . j}=L t_{i} & +L b_{j}+L(t b)_{i j}+L_{k}+L\left(t^{\prime} b\right)_{k j}+ \\
& +L t_{i k}^{\prime}+L\left(t t^{\prime} b\right)_{i k j}+\sum_{\ell} e_{i k \ell j},
\end{aligned}
$$

então:

$$
\begin{aligned}
\frac{1}{L} E\left(\sum_{i j k} y_{i k \cdot j}^{2}\right)= & J K L \sum_{i} t_{i}^{2}+I J K L \sigma_{b}^{2}+I J K L \sigma_{t b}^{2}+ \\
& +I J L \sum_{k} t_{k}^{2}+I J K L \sigma_{t^{\prime} b}^{2}+ \\
& +J L \sum_{i k}\left(t t^{\prime}\right){ }_{i k}^{2}+I J K L \sigma_{t t^{\prime} b}^{2}+I J K \sigma^{2}
\end{aligned}
$$




$$
\text { Subtrindo-se a } E(C), E(S Q T), E(S Q B), E\left(S Q T^{\prime}\right) \text {, }
$$

$E\left(S Q T \times T^{\prime}\right), E[S Q R(s)]$ e a $E[S Q R(b)]$, vem:

$$
\begin{aligned}
\operatorname{E[SQR}(\mathrm{c})]= & \mathrm{L}(\mathrm{I}-1)(\mathrm{J}-1)(\mathrm{K}-1) \sigma_{\mathrm{t} \mathrm{t}^{\prime} \mathrm{b}}^{2}+ \\
& +(\mathrm{I}-1)(\mathrm{J}-1)(\mathrm{K}-1) \sigma^{2},
\end{aligned}
$$

dividindo-se por $(I-1)(K-1)(J-1)$ :

$$
E[Q M \operatorname{Residuo(c)}]=L \sigma_{t t^{\prime} b}^{2}+\sigma^{2} \text {. }
$$

i) Analogamente ao caso anterior, $Q M^{*} *=\frac{S Q T *}{L-1}$, então:

$$
\mathrm{E}(\mathrm{QMT} *)=\frac{1}{(\mathrm{~L}-1)} \mathrm{E}(\mathrm{SQT} *)
$$

onde:

$$
\mathrm{SQT} *=\frac{1}{\mathrm{IJK}} \sum_{\ell} \mathrm{T}_{\ell}^{2}-\mathrm{C}
$$

e

$$
\begin{aligned}
T *=I K \sum_{j} b_{j} & +K \sum_{i j}(t b)_{i j}+I \sum_{j k}\left(t^{\prime} b\right)_{k j}+ \\
& +\sum_{i j k}\left(t t^{\prime} b\right)_{i k j}+I J K t_{l}^{*}+\sum_{i j k} e_{i k \ell j},
\end{aligned}
$$

então:

$$
\begin{aligned}
\frac{1}{I J K} E\left(\sum T_{i}^{2}\right)=I K L \sigma_{b}^{2} & +K L \sigma_{t b}^{2}+I L \sigma_{t}^{2} b+L \sigma_{t}^{2} t^{\prime} b+ \\
& +I J K \underset{l}{\sum} t_{l}^{\frac{2}{k}}+L \sigma^{2}
\end{aligned}
$$




$$
\begin{aligned}
& \text { Subtraindo-se a } E(C) \text {, vem: } \\
& E\left(S_{T} *\right)=\operatorname{IJK} \underset{l}{\sum_{l} t \frac{2}{*}+(L-1) \sigma^{2},}
\end{aligned}
$$

dividindo-se por $(L-1)$ :

$$
E(Q M T *)=\frac{I J K}{(L-1)} \sum_{\ell} t_{l}^{*}+\sigma^{2} .
$$

j) Analogamente ao caso anterior; QMTxT* $=\frac{\mathrm{SQTxT} *}{(\mathrm{I}-1)(\mathrm{L}-1)}$, então:

$$
E(\operatorname{QMTxT} *)=\frac{1}{(I-1)(L-1)} E(\operatorname{SQTxT} *)
$$

onde:

$$
\operatorname{SQTxT*}=\frac{1}{\mathrm{JK}} \underset{i \ell}{\sum}\left(\mathrm{TT}_{\mathrm{i} \ell}^{*}\right)^{2}-\mathrm{C}-\mathrm{SQT}-\mathrm{SQT} *
$$

e

$$
\begin{aligned}
& T T^{*}=J K t_{i}+K \sum_{j} b_{j}+K \sum_{j}(t b)_{i j}+\underset{j k}{\sum}\left(t^{\prime} b\right)_{k j}+ \\
& +\sum_{j k}\left(t t^{\prime} b\right)_{i k j}+J K t_{\ell}^{*}+J K t t_{i \ell}^{*}+ \\
& +\sum_{j k} e_{i k l j},
\end{aligned}
$$

então:

$$
\begin{aligned}
\frac{1}{\mathrm{JK}} \mathrm{E}\left[\sum_{i \ell}(\mathrm{TT} *)_{i \ell}^{2}\right]= & \mathrm{JKL} \sum_{i} \mathrm{t}_{i}^{2}+\mathrm{IKL} \sigma_{\mathrm{b}}^{2}+\mathrm{IKL} \sigma_{\mathrm{tb}}^{2}+ \\
& +\mathrm{IL} \sigma_{t^{\prime} \mathrm{b}}^{2}+\mathrm{IL} \sigma_{\mathrm{t} \mathrm{t}^{\prime} \mathrm{b}}^{2}+\mathrm{IJK} \sum_{\ell} \mathrm{t}_{\ell}^{\frac{2}{*}}+ \\
& +\mathrm{JK} \sum_{i \ell}(\mathrm{tt} *)_{i \ell}^{2}+\mathrm{IL} \sigma^{2} .
\end{aligned}
$$


Subtraindo-se a $E(C), E(S Q T)$ e E(SQT*), vem:

$$
\left.\mathrm{E}(\mathrm{SQT} \times \mathrm{T} *)=\mathrm{JK} \sum_{i \ell}(\mathrm{t} t *)\right)_{i \ell}^{2}+(\mathrm{I}-1)(\mathrm{L}-1) \sigma^{2},
$$

dividindo-se por $(I-1)(L-1)$ :

$$
E(Q \operatorname{MTxT} *)=\frac{\mathrm{JK}}{(\mathrm{I}-1)(\mathrm{L}-1)} \sum_{i \ell}\left(t t^{*}\right)_{i \ell}^{2}+\sigma^{2} .
$$

k) Partindo-se de $\mathrm{QMT}^{\prime} \mathrm{xT}^{*}=\frac{\mathrm{SQT}^{\prime} \mathrm{xT} \mathrm{T}^{*}}{(\mathrm{~K}-1)(\mathrm{L}-1)}$, tem-se:

$$
E\left(Q M T^{\prime} x T^{*}\right)=\frac{1}{(K-1)(L-1)} E\left(S Q T T^{\prime} \times T^{*}\right),
$$

onde:

$$
\mathrm{SQT}^{\prime} \mathrm{xT} *=\frac{1}{\mathrm{IJ}} \sum_{\mathrm{k} \ell}\left(\mathrm{T}^{\prime} \mathrm{T}^{*}\right)_{\mathrm{k} \ell}^{2}-\mathrm{C}-\mathrm{SQT} \mathrm{T}^{\prime}-\mathrm{SQT} *
$$

e

$$
\begin{aligned}
T^{\prime} T *=I \sum_{j} b_{j} & +I J t_{k}^{\prime}+I J t_{l}^{*}+I J\left(t^{\prime} t^{*}\right)_{k \ell}+ \\
& +\sum_{i j}(t b)_{i j}+I \sum_{j}\left(t^{\prime} b\right)_{k j}+ \\
& +\sum_{i j}\left(t t^{\prime} b\right)_{i k j}+\sum_{i j} e_{i k \ell j},
\end{aligned}
$$

então:

$$
\begin{aligned}
\frac{1}{I J} E\left[\sum_{k l}\left(T^{\prime} T^{*}\right){ }_{k l}^{2}\right]= & I K L \sigma_{b}^{2}+I J L \sum_{k} t_{k}^{2}+I J K \sum_{\ell} t_{l}^{\frac{2}{*}}+ \\
& +I J \sum_{k l}\left(t^{\prime} t *\right){ }_{k l}^{2}+K L \sigma_{t b}^{2}+ \\
& +I K L \sigma_{t^{\prime} b}^{2}+K L \sigma_{t t^{\prime} b}^{2}+K L \sigma^{2} .
\end{aligned}
$$




$$
\begin{aligned}
& \text { Subtraindo-se a } E(C), E\left(S Q T^{\prime}\right) \text { e } E(S Q T *) \text {, vem: } \\
& E\left(S Q T^{\prime} \times T^{*}\right)=I J \sum_{k \ell}^{\sum}\left(t^{\prime} t *\right){ }_{k \ell}^{2}+(K-1)(L-1) \sigma^{2}
\end{aligned}
$$

dividindo-se por $(K-1)(L-1)$ :

$$
E\left(Q M T^{\prime} \mathrm{T} *\right)=\frac{\mathrm{IJ}}{(\mathrm{K}-1)(\mathrm{L}-1)} \sum_{\mathrm{k} l}\left(\mathrm{t}^{\prime} \mathrm{t} *\right)_{\mathrm{k} l}^{2}+\sigma^{2}
$$

1) Partindo-se de $\mathrm{QMTxT}^{\prime} \mathrm{MT}^{*}=\frac{\mathrm{SQT}^{*} \mathrm{~T}^{\prime} \mathrm{xT} *}{(\mathrm{I}-1)(\mathrm{K}-1)(\mathrm{L}-1)}$, tem-se:

$$
\mathrm{E}\left(\mathrm{QMT} \times \mathrm{T}^{\prime} \times \mathrm{T} *\right)=\frac{1}{(\mathrm{I}-1)(\mathrm{K}-1)(\mathrm{L}-1)} \mathrm{E}\left(\mathrm{SQTx} \mathrm{T}^{\prime} \times \mathrm{T} *\right)
$$

ond e:

$$
\begin{aligned}
& \operatorname{SQT\times T} \mathrm{T}^{\prime} \mathrm{TT} *=\frac{1}{\mathrm{~J}} \sum_{i \mathrm{kl}}\left(\mathrm{TT}^{\prime} \mathrm{T} *\right)_{i \mathrm{kl}}^{2}-\mathrm{C}-\mathrm{SQT}-\mathrm{SQT} \mathrm{T}^{\prime}- \\
& -\mathrm{SQT}^{*}-\mathrm{SQT}^{\prime} \mathrm{T}^{\prime}-\mathrm{SQT} \times \mathrm{T} *-\mathrm{SQT}^{\prime} \times \mathrm{T} *
\end{aligned}
$$

e

$$
\begin{aligned}
& \mathrm{TT}^{\prime} \mathrm{T}^{*}=\mathrm{J} \mathrm{t}_{i}+\sum_{j} \mathrm{~b}_{j}+\sum_{j}(\mathrm{tb})_{i j}+\mathrm{J}_{\mathrm{j}}+\mathrm{t}_{j}^{\prime}\left(\mathrm{t}^{\prime} \mathrm{b}\right)_{\mathrm{k} j} \\
& +J\left(t t^{\prime}\right)_{i k}+\sum_{j}\left(t t^{\prime} b\right)_{i k}+J+\frac{t}{\ell}+ \\
& +J\left(t t^{*}\right)_{i \ell}+J\left(t^{\prime} t^{*}\right)_{k \ell}+J\left(t t^{\prime} t *\right)_{i k \ell}+ \\
& +\sum_{j} e_{i k \ell j,}
\end{aligned}
$$

ent ão: 


$$
\begin{aligned}
& \frac{1}{J} E\left[\underset{i k \ell}{\sum_{i k}}\left(\mathrm{TT}^{\prime} \mathrm{T}^{*}\right)_{i \mathrm{k} \ell}^{2}\right]=\mathrm{JKL} \underset{i}{\sum_{i}} \mathrm{t}_{i}^{2}+\mathrm{IJKL} \sigma_{\mathrm{b}}^{2}+I K L \sigma_{\mathrm{tb}}^{2}+ \\
& +\operatorname{IJL} \sum_{k} t_{k}^{2}+I J K L \sigma_{t}^{2} b^{+}+ \\
& +J L \sum_{i k}\left(t t^{\prime}\right) \underset{i k}{2}+I K L \sigma_{t t^{\prime} b}^{2}+ \\
& +\operatorname{IJK} \underset{\ell}{\sum} t_{\ell}^{2}+\mathrm{JK} \underset{i \ell}{\sum}(\mathrm{t} *) \underset{i \ell}{2}+ \\
& +\operatorname{IJ} \sum_{k \ell}\left(t^{\prime} t *\right) \underset{k \ell}{2}+ \\
& +\mathrm{J} \sum_{i k \ell}\left(t t^{\prime} t *\right) \underset{i k \ell}{2}+I K L \sigma^{2} .
\end{aligned}
$$

Subtraindo-se a $E(C), E(S Q T), E\left(S Q T^{\prime}\right), E\left(S Q T^{*}\right)$, $E\left(S Q T x T^{\prime}\right), E\left(S Q T x T^{*}\right)$ e a $E\left(S Q T^{\prime} x T^{*}\right)$, vem:

$$
\mathrm{E}\left(\mathrm{SQT \times T} \mathrm{T}^{\prime} \mathrm{T} *\right)=\mathrm{J} \sum_{\mathrm{ik \ell}}\left(\mathrm{t} \mathrm{t}^{\prime} \mathrm{t} *\right) \underset{\mathrm{i} k \ell}{2}+(\mathrm{I}-1)(\mathrm{K}-1)(\mathrm{L}-1) \sigma^{2}
$$

dividindo-se por $(\mathrm{I}-1)(\mathrm{K}-1)(\mathrm{L}-1)$,

$$
\mathrm{E}\left[\mathrm{QMT} \times \mathrm{T}^{\prime} \mathrm{xT} \mathrm{T}^{*}\right]=\frac{\mathrm{J}}{(\mathrm{I}-1)(\mathrm{K}-1)(\mathrm{L}-1)} \underset{\mathrm{ik} \ell}{\sum}\left(\mathrm{t} \mathrm{t}^{\prime} \mathrm{t} *\right)_{\mathrm{ik} \ell}^{2}+\sigma^{2}
$$

m) Partindo-se de $Q M R(d)=\frac{S Q R(d)}{I K(J-1)(L-1)}$, tem-se:

$$
\operatorname{E}[\operatorname{QMR}(d)]=\frac{1}{I K(J-1)(L-1)} \operatorname{E}[\operatorname{SQR}(d)],
$$

ond e :

$$
\begin{gathered}
\operatorname{SQR}(d)=\sum_{i j k \ell} y_{i k \ell j}^{2}-\frac{1}{L} \sum_{i j k} y_{i k . j}^{2}-S Q T *-S Q T x T *- \\
-S Q T T^{\prime} T^{*}-S Q T x T ' x T *
\end{gathered}
$$


e

$$
\begin{aligned}
y_{i k \ell j}=t_{i} & +b_{j}+(t b)_{i j}+t_{k}^{\prime}+\left(t^{\prime} b\right)_{k j}+t i_{i k}^{\prime} \\
& +\left(t t^{\prime} b\right)_{i k j}+t_{\ell}^{*}+t i_{i \ell}^{*}+t^{\prime} t k_{k \ell}^{*}+t t^{\prime} \underbrace{*}_{i k \ell}+ \\
& +e_{i k \ell j},
\end{aligned}
$$

então:

$$
\begin{aligned}
& \sum_{i j k \ell} E\left(y_{i k j \ell}^{2}\right)=J K L \sum_{i} t_{i}^{2}+I J K L \sigma_{b}^{2}+I J K L \sigma \underset{t b}{2}+ \\
& +\operatorname{IJL} \sum_{k} t_{k}^{2}+I J K L \sigma_{t^{\prime} b}^{2}+J L \sum_{i k}\left(t t^{\prime}\right) \underset{i k}{2}+
\end{aligned}
$$

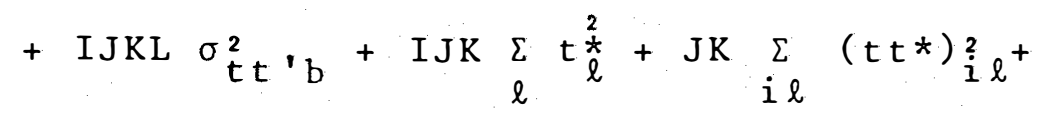

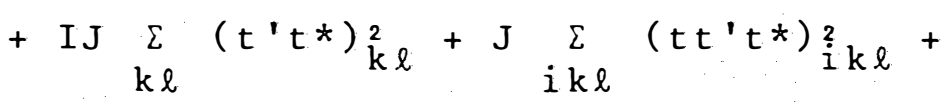

$$
\begin{aligned}
& + \text { IJKL } \sigma^{2}
\end{aligned}
$$

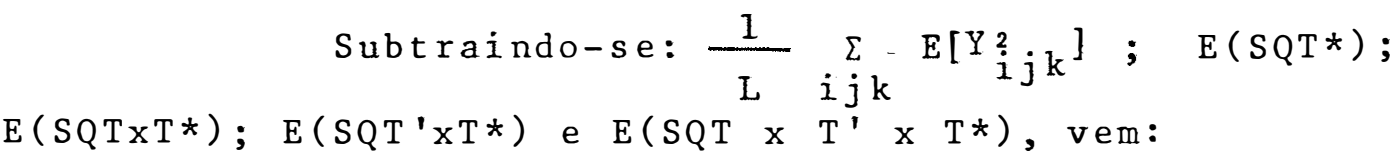

$$
E[S Q R \cdot(d)]=I K(J-1)(L-I) \sigma^{2}, \text {, }
$$

dividindo-se por IK $(J-1)(L-1)$, tem-se:

$$
\operatorname{E}[\mathrm{QMR}(\mathrm{d})]=\sigma^{2} \text {. }
$$


3.3 .3 - Testes de hipōteses e quadro de anālise de variāncia

Segundo KEMPTHORNE (1952), CHAKRABARTI (1962)

e PIMENTEL GOMES (1976), as hipóteses de nulidade usuais, nos experimentos em faixas, são geralmente:

$$
\begin{array}{llll}
\mathrm{H}_{\mathrm{O}}(1): & \mathrm{t}_{\mathrm{i}}=0 & ; & \mathrm{i}=1,2, \ldots, \mathrm{I} ; \\
\mathrm{H}_{\mathrm{O}}(2): & \mathrm{t}_{\mathrm{k}}^{\prime}=0 & \mathrm{k} & =1,2, \ldots, \mathrm{K} ; \\
\mathrm{H}_{\mathrm{O}}(3): \mathrm{t}_{\mathrm{ik}}^{\prime}=0 & ; & \mathrm{i} & =1,2, \ldots, \mathrm{I}, \\
\mathrm{k} & =1,2, \ldots, \mathrm{K} ;
\end{array}
$$

representando, respectivamente, as hipóteses de nulidade para efeitos $T, T^{\prime}$ e para efeito da interação $T \times T^{\prime}$. Dados os objetivos deste estudo, a essas hipóteses foram acrescentadas:

$$
\begin{aligned}
& \mathrm{H}_{\mathrm{O}}(4): \quad \mathrm{t}_{\ell}^{*}=0 \quad ; \quad l=1,2, \ldots, \mathrm{L} \text {; }
\end{aligned}
$$

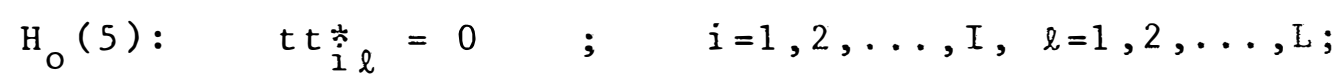

$$
\begin{aligned}
& \mathrm{H}_{\mathrm{O}}(6): \quad \mathrm{t}^{\prime} \mathrm{t}_{\mathrm{k} \ell}^{*}=0 \quad ; \quad \mathrm{k}=1,2, \ldots, \mathrm{K}, \ell=1,2, \ldots, \mathrm{L} \text {; } \\
& \mathrm{H}_{\mathrm{O}}(7): \mathrm{t}^{\prime} \mathrm{t}_{\mathrm{ik \ell}}=0 \quad ; \quad \mathrm{i}=1,2, \ldots, \mathrm{I}, \mathrm{k}=1,2, \ldots, \mathrm{K}, \\
& \ell=1,2, \ldots, \mathrm{L} \text {; }
\end{aligned}
$$

que correspondem respectivamente às hipóteses de nulidade para efeito do tratamento $T^{*}$, das interações $T \times T *$ e $T^{\prime} T^{*}$ e para efeito da interação tripla entre os trēs tipos de trat amentos. 
Essas hipóteses adicionais estão coerentes com aquelas sugeridas em HEDAYETULLAH et $a$ li $i$ (1944) que embora sem discussões ou 'demonstrações teóricas, apresentaram um quadro para a anālise de variāncia de um experimento com três 1 inhagens de "arroz de inverno", seis datas de plantio e très espaçamentos.

Aqui, os residuos adequados para os testes de hipótese ficaram evidentes, quando se observou a coluna relativa às esperanças dos quadrados médios, conforme o quadro 8 . 

Quadro 8 - Esquema de anālise de variancia e esperanças dos quadra
dos médios, para experimentos em faixas com subparcelas
subdivididas.

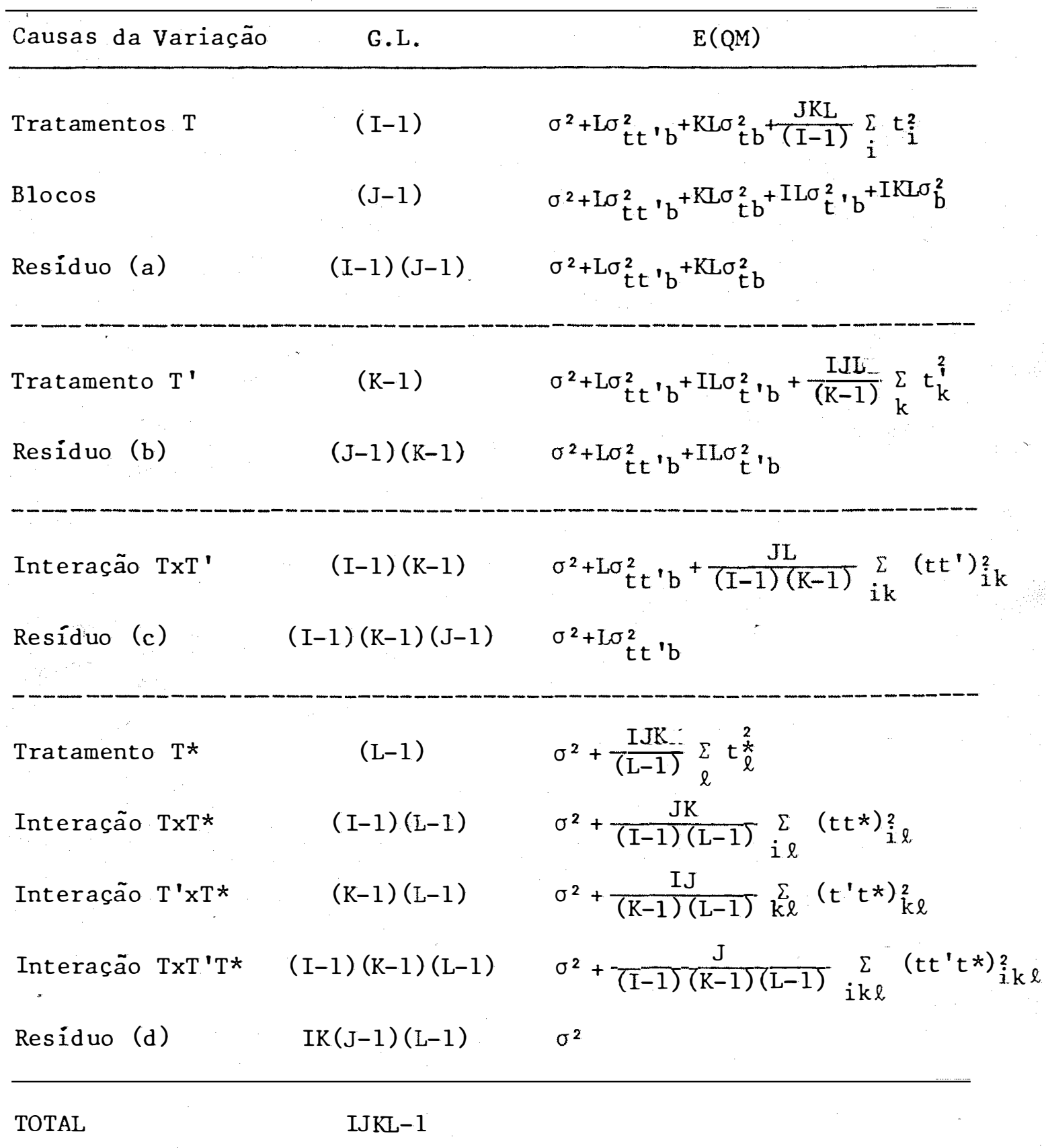


Assim, quando os dados são balanceados, como sugerido nas deduções aqui ef etuadas, ficam bem determinados os critérios para os respectivos testes, conforme se verifica no quadro 9.

Quadro 9 - Critérios para os testes de hipóteses.

\begin{tabular}{|c|c|c|}
\hline Hi pót es es & G.I. & Valores de F \\
\hline $\mathrm{H}_{\mathrm{O}}(1)$ & $(I-1) ;(I-1)(J-1)$ & {$[\mathrm{QMT}] /[\mathrm{QMRes}(\mathrm{a})]$} \\
\hline $\mathrm{H}_{\mathrm{O}}(2)$ & $(\mathrm{K}-1) ;(\mathrm{J}-1)(\mathrm{K}-1)$ & {$\left[\mathrm{QMT}^{\prime}\right] /[\mathrm{QMRes}(\mathrm{b})]$} \\
\hline $\mathrm{H}_{\mathrm{O}}(3)$ & $(\mathrm{I}-1)(\mathrm{K}-1) ;(\mathrm{I}-1)(\mathrm{J}-1)(\mathrm{K}-1)$ & {$\left[\mathrm{QMTxT}^{\prime}\right] /[\mathrm{QMRes}(\mathrm{c})]$} \\
\hline $\mathrm{H}_{\mathrm{O}}(4)$ & $(\mathrm{L}-1) ; \operatorname{IK}(\mathrm{J}-1)(\mathrm{L}-1)$ & {$[\mathrm{QMT} *] /[\mathrm{QMRes}(\mathrm{d})]$} \\
\hline $\mathrm{H}_{\mathrm{O}}(5)$ & $(\mathrm{I}-1)(\mathrm{L}-1) ; \mathrm{IK}(\mathrm{J}-1)(\mathrm{L}-1)$ & {$[\mathrm{QMTxT} *] /[\mathrm{QMRes}(\mathrm{d})]$} \\
\hline $\mathrm{H}_{\mathrm{O}}(6)$ & $(\mathrm{K}-1)(\mathrm{L}-1) ; \operatorname{IK}(\mathrm{J}-1)(\mathrm{L}-1)$ & {$\left[\mathrm{QMT}^{\prime} \mathrm{xT} *\right] /[\mathrm{QMRes}(\mathrm{d})]$} \\
\hline $\mathrm{H}_{\mathrm{O}}(7)$ & $(\mathrm{I}-1)(\mathrm{K}-1)(\mathrm{L}-1) ; \mathrm{IK}(\mathrm{J}-1)(\mathrm{L}-1)$ & [QMTxT 'xT*]/[QMRes (d)] \\
\hline
\end{tabular}

Além das estruturas apresentadas para os tes tes de hipóteses, poderiam ser considerados os casos refe rentes aos desdobramentos das interações tais como: niveis de T num mesmo nivel de $T^{\prime}$, e outros anälogos. Entre tanto, todos esses casos serão abordados no método de comparações mültiplas descrito a seguir. 


\section{4 - Variāncias de Contrastes}

Considerando-se a possibilidade de rejeição de ao menos uma das hipóteses de nulidade, formularam-se as regras para os testes entre as estimativas dos efeitos dos parāmetros envolvidos.

Para tanto, foram obtidas as estimativas das variancias das estimativas dos doze tipos possiveis decon trastes entre duas mëdias de tratamentos, conforme demons trado a seguir.

a) Diferença entre duas médias de tratamentos T:

$$
\begin{aligned}
& P_{i}=\overline{\mathrm{m}}_{i}-\hat{\mathrm{m}}_{i} \\
& \text { Dado que } \mathrm{T}_{i}=\sum_{j \mathrm{kl}} \mathrm{y}_{\mathrm{ik \ell j}} \text { e usando-se as res- } \\
& \text { trições citadas em }(3.2 .1) \text {, vem: } \\
& T_{i}=J K L t_{i}+K L \sum_{j} b_{j}+K L \sum_{j}(t b)_{i j}+L \sum_{k j}\left(t^{\prime} b\right)_{k j}+ \\
& +L \sum_{k j}\left(t t^{\prime} b\right)_{i k j}+\underset{j k \ell}{\sum} e_{i k \ell j} \\
& \hat{\mathrm{m}}_{i}=\frac{\mathrm{T}_{i}}{\mathrm{JKL}}=\mathrm{t}_{i}+\frac{1}{J} \sum_{j} b_{j}+\frac{1}{J} \sum_{j}(t b)_{i j}+\frac{1}{J K} \sum_{k j}\left(t^{\prime} b\right)_{k j}+ \\
& +\frac{1}{J K} \sum_{j k}\left(t t^{\prime} b\right)_{i k j}+\frac{1}{J K L} \sum_{j k \ell} e_{i k \ell j} .
\end{aligned}
$$




\section{Por analogia:}

$$
\begin{aligned}
& \tilde{m}_{i}=t_{i},+\frac{1}{J} \sum_{j} b_{j}+\frac{1}{J} \sum_{j}(t b)_{i}{ }_{j}+\frac{1}{J k} \sum_{k j}(t)^{\prime} b j+ \\
& +\frac{1}{J K} \sum_{j k}\left(t t^{\prime} b\right)_{i}{ }^{\prime} k j+\frac{1}{J K L} \sum_{j k \ell} e_{i}^{\prime} j k \ell \\
& \hat{P}_{i}=t_{i}-t_{i}{ }^{\prime}+\frac{1}{J} \sum_{j}\left(t b_{i j}-t b_{i}{ }^{\prime}\right)^{\prime}+ \\
& +\frac{1}{J K} \sum_{k j}\left(t t^{\prime} b_{i k j}-t t^{\prime} b_{i} k_{j}\right)+ \\
& +\frac{1}{J K L} \sum_{j k \ell}\left(e_{i k \ell j}-e_{i}{ }^{\prime} k \ell j\right) \text {, }
\end{aligned}
$$

e então:

$$
E\left(\bar{Y}_{i}\right)=t_{i}-t_{i} \text {, }
$$

Por outro lado,

$$
V\left(\hat{Y}_{i}\right)=E\left[\hat{Y}_{i}-E\left(\hat{Y}_{i}\right)\right]^{2}
$$

substituindo-se tem-se:

$$
\begin{aligned}
& V\left(\hat{Y}_{i}\right)=E\left[\begin{array}{r}
\left.\frac{1}{J} \sum_{j}\left(t b_{i j}-t b_{i}, .\right)+\frac{1}{J K} \sum_{j k}\left(t t^{\prime} b_{i k j}-t t^{\prime} b_{i}{ }^{\prime} k j\right)+\right]^{2} \\
+\frac{1}{J K L} \sum_{j k \ell}\left(e_{i k j \ell}-e_{i}{ }^{\prime} k \ell j\right)
\end{array}\right]^{2} \\
& V\left(\hat{Y}_{i}\right)=\frac{1}{J^{2}} 2 J \sigma_{t b}^{2}+\frac{1}{J^{2} \mathrm{~K}^{2}} 2 \mathrm{KJ} \sigma_{t t^{\prime} b}^{2}+\frac{1}{J^{2} \mathrm{~K}^{2} \mathrm{~L}^{2}} 2 \mathrm{JKL} \sigma^{2} \\
& V\left(\hat{Y}_{i}\right)=\frac{2}{J K L}\left(K L \sigma_{t b}^{2}+L \sigma_{t}^{2} t_{b}^{\prime}+\sigma^{2}\right)
\end{aligned}
$$


sua estimativa:

$$
\hat{V}\left(\hat{Y}_{i}\right)=\frac{2}{J K L}[Q M \operatorname{Residuo}(a)],
$$

que è um resultado concordante com aqueles encontrados em CHAKRABART I (1962), FEDERER (1955), COCHRAN e COX (1976)

e ZIMMERMANN (1973) para a mesma variância quando o ensaio em faixas, com dois tratamentos, é delineado em blocos ca sualizados.

b) Diferença entre duas médias de tratamentos $T^{\prime}$ :

$$
\hat{\mathrm{Y}}_{\mathrm{k}}=\hat{\mathrm{m}}_{\mathrm{k}}-\hat{\mathrm{m}}_{\mathrm{k}},
$$

Dado que:

$$
\mathrm{T}_{\mathrm{k}}^{\prime}=\sum_{\mathrm{ij \ell}} \mathrm{y}_{\mathrm{ik} \ell \mathrm{j}}
$$

e de acordo com as condições anteriores, vem:

$$
\begin{gathered}
T_{k}^{\prime}=I L \sum_{j} b_{j}+I J L t_{k}^{\prime}+L \sum_{i j}(t b)_{i j}+I L \sum_{j}\left(t^{\prime} b\right)_{k j}+ \\
+L \sum_{i j}\left(t t^{\prime} b\right)_{i k j}+\sum_{i j l} e_{i k l j} \\
\bar{m}_{k}=\frac{t_{k}^{\prime}}{I J L}=\frac{1}{J} \sum_{j} b_{j}+t_{k}^{\prime}+\frac{1}{I J} \sum_{i j}(t b)_{i j}+\frac{1}{J} \sum_{j}\left(t^{\prime} b\right)_{k j}+ \\
\quad+\frac{1}{I J} \sum_{i j}\left(t t^{\prime} b\right)_{i k j}+\frac{1}{I J L} \sum_{i j l} e_{i k l j} .
\end{gathered}
$$




\section{Por analogia:}

$$
\begin{aligned}
& \hat{\mathrm{m}}_{k^{\prime}}=\frac{1}{J} \sum_{j} b_{j}+t_{k^{\prime}}^{\prime}+\frac{1}{I J} \sum_{i j}(t b)_{i j}+\frac{1}{J} \sum_{j}\left(t^{\prime} b\right)_{k^{\prime} j}+ \\
& +\frac{1}{I J} \sum_{i j}\left(t t^{\prime} b\right)_{i k^{\prime} j}+\frac{1}{I J L} \sum_{i j \ell} e_{i k} k^{\prime} j \\
& \widehat{Y}_{k}=t^{\prime}{ }_{k}-t_{k^{\prime}}^{\prime}+\frac{1}{J} \sum_{i}\left(t^{\prime} b_{k j}-t^{\prime} b_{k}{ }^{\prime} j^{\prime}+\right. \\
& +\frac{1}{I J} \sum_{i j}\left(t t^{\prime} b_{i k j}-t t^{\prime} b_{i k}{ }^{\prime}\right)+ \\
& +\frac{1}{I J L} \sum_{i j \ell}\left(e_{i k \ell j}-e_{i k \ell \ell j}\right) \text {, }
\end{aligned}
$$

então:

$$
\begin{aligned}
& E\left(\bar{Y}_{k}\right)=t_{k}^{\prime}-t_{k^{\prime}}^{\prime} . \\
& \text { Por outro lado: } \\
& V\left(\widehat{Y}_{k}\right)=E\left[\widehat{Y}_{k}-E\left(\widehat{Y}_{k}\right)\right]^{2}, \\
& \because V\left(\bar{Y}_{k}\right)=E\left[\begin{array}{l}
\frac{1}{J} \sum_{j}\left(t^{\prime} b_{k j}-t^{\prime} b_{k}{ }^{\prime}\right)+ \\
+\frac{1}{I J} \sum_{i j}\left(t t^{\prime} b_{i k j}-t t^{\prime} b_{i k}{ }^{\prime}\right)+ \\
+\frac{1}{I J L} \sum_{i j l}\left(e_{i k \ell j}-e_{i k}{ }^{\prime} \ell j\right.
\end{array}\right]^{2} \\
& \therefore V\left(\bar{Y}_{k}\right)=\frac{2}{I J L}\left(I L \sigma_{t^{\prime} b}^{2}+L \sigma_{t t^{\prime} b}^{2}+\sigma^{2}\right) \text {, }
\end{aligned}
$$


e sua estimativa será:

$$
\nabla\left(\widehat{Y}_{k}\right)=\frac{2}{\text { IJL }}[Q M R(b)],
$$

que é um resultado concordante com aqueles encontrados em CHAKRABART I (1962), FEDERER (1955), COCHRAN e COX (1976)

e ZIMMERMANN (1973), para a mesma variāncia quando o ensaio em faixas com dois tratamentos é delineado em blocos casualizados.

c) Diferença entre duas médias de tratamentos $\mathrm{T}$ *:

$$
\bar{P}_{\ell}=\overline{\mathrm{m}}_{\ell}-\overline{\mathrm{m}}_{\ell}
$$

Dado que $T_{\ell}^{*}=\sum_{i j k} y_{i k l j}$ ou, de acordo com as condições anteriores:

$$
\begin{aligned}
& T_{\ell}^{*}=I K \sum_{j} b_{j}+K \sum_{i j}(t b)_{i j}+I \sum_{j k}\left(t^{\prime} b\right)_{k j}+ \\
& +\sum_{i j k}\left(t t^{\prime} b\right) i k j+I J K{ }_{\ell}^{*}+\sum_{i j k} e_{i k l j} \\
& \hat{\mathrm{m}}_{\ell}=\frac{\mathrm{T}_{\ell}^{*}}{I J K}=\frac{1}{J} \sum_{j} b_{j}+\frac{1}{I J} \sum_{i j}(t b)_{i j}+\frac{1}{J K} \sum_{j k}\left(t^{\prime} b\right)_{k j}+ \\
& +\frac{1}{I J K} \sum_{i j k}\left(t t^{\prime} b\right)_{i k j}+t / t_{l}^{*}+\frac{1}{I J K} \sum_{j k} e_{i k l j} .
\end{aligned}
$$

Por analogia: 
61.

$$
\begin{aligned}
\hat{\mathrm{m}}_{l^{\prime}}=\frac{1}{J} \sum_{j} b_{j} & +\frac{1}{I J} \sum_{i j}(t b)_{i j}+\frac{1}{J K} \sum_{j k}\left(t^{\prime} b\right)_{k j}+ \\
& +\frac{1}{I J K} \sum_{i j k}\left(t t^{\prime} b\right)_{i k j}+t_{l^{\prime}}+\frac{1}{I J K} \sum_{i j k} e_{i k \ell^{\prime} j}
\end{aligned}
$$$$
E\left(\hat{Y}_{\ell}\right)=t_{\ell}^{*}-t_{\ell}^{*}
$$$$
V\left(\hat{Q}_{\ell}\right)=E\left[\frac{1}{I J K} \sum_{i j k}\left(e_{i k \ell j}-e_{i k \ell^{\prime} j}\right)\right]^{2}
$$

$$
\begin{aligned}
\therefore \quad V\left(\hat{Y}_{\ell}\right) & =\frac{1}{I^{2} J^{2} K^{2}} 2 I J K \sigma^{2} \\
V\left(\hat{Y}_{\ell}\right) & =\frac{2}{I J K} \sigma^{2}
\end{aligned}
$$

e sua estimativa

$$
\hat{V}\left(\hat{\mathrm{Y}}_{\ell}\right)=\frac{2}{\mathrm{IJK}}[\mathrm{QMR}(\mathrm{d})]
$$

d) Diferença entre duas médias de tratamentos $T$, dentro de um mesmo nivel de tratamento $T^{\prime}$ :

$$
\hat{\mathrm{Y}}_{i / k}=\hat{\mathrm{m}}_{i k}-\hat{\mathrm{m}}_{i}{ }^{\prime k} \text {. }
$$

Dado que:

$$
\mathrm{T}_{i} \mathrm{~T}_{\mathrm{k}}^{\prime}=\sum_{j \ell} \mathrm{y}_{\mathrm{ik \ell j}}
$$

ent ão: 


$$
\begin{aligned}
\mathrm{T}_{i} \mathrm{~T}_{k}^{\prime}=J L t_{i} & +L_{j} \mathrm{~b}_{j}+\sum_{j}(t b)_{i j}+J L t_{k}^{\prime}+\sum_{j}\left(t^{\prime} b\right)_{k j}+ \\
& +J L\left(t t^{\prime}\right)_{i k}+\sum_{j}^{\sum}\left(t t^{\prime} b\right)_{i k j}+\sum_{j \ell} e_{i k \ell j},
\end{aligned}
$$

donde:

$$
\begin{aligned}
\hat{\mathrm{m}}_{i k}=\frac{\mathrm{T}_{i}{ }^{T} k^{\prime}}{\mathrm{JL}}=t_{i} & +\frac{1}{\mathrm{~J}} \sum_{j} b_{j}+\frac{1}{J} \sum_{j}(t b)_{i j}+t_{k}^{\prime}+ \\
& +\frac{1}{J} \sum_{j}\left(t^{\prime} b\right)_{k j}+t t_{i k}^{\prime}+\frac{1}{J} \sum_{j}\left(t t^{\prime} b\right)_{i k j}+ \\
& +\frac{1}{J L} \sum_{j \ell} e_{i k \ell j} .
\end{aligned}
$$

Por analogia:

$$
\begin{aligned}
& \overline{\mathrm{m}}_{\mathrm{i}^{\prime} \mathrm{k}}=\mathrm{t}_{\mathrm{i}^{\prime}}+\frac{1}{\mathrm{~J}} \sum_{j} \mathrm{~b}_{j}+\frac{1}{\mathrm{~J}}-\sum_{j}(t b)_{i}{ }^{\prime} j+t_{k}^{\prime}+ \\
& +\frac{1}{J} \sum_{j}\left(t^{\prime} b\right)_{k j}+t t_{i ! k}^{\prime}+\frac{1}{J} \sum_{j}\left(t t^{\prime} b\right)_{i \cdot k j}+ \\
& +\frac{1}{J L} \sum_{j \ell} e_{i}^{\prime} k \ell j \\
& \hat{Y}_{i / k}=t_{i}-t_{i,}{ }^{\prime}+\frac{1}{J} \sum_{j}\left[(t b)_{i j}-(t b)_{i}{ }^{\prime}\right]+ \\
& +\left(t t_{i k}^{\prime}-t t_{i}^{\prime} k^{\prime}\right)+\frac{1}{J} \sum_{j}\left[\left(t t^{\prime} b\right)_{i k j}-\left(t t^{\prime} b\right)_{i}{ }^{\prime} k j\right]+ \\
& +\frac{1}{J L} \sum_{j \ell}\left(e_{i k \ell j}-e_{i ' k \ell j}\right)
\end{aligned}
$$

então:

$$
E\left(\hat{Y}_{i / k}\right)=\left(t_{i}-t_{i}{ }^{\prime}\right)+\left(t t_{i k}-t t_{i}{ }^{\prime}{ }_{k}\right)
$$


e

$$
\begin{aligned}
& V\left(\hat{Y}_{i / k}\right)=E\left[\begin{array}{l}
\frac{1}{J} \sum_{j}\left(t b_{i j}-t b_{i j}\right)+ \\
+\frac{1}{J} \sum_{j}\left(t t^{\prime} b_{i k j}-t t^{\prime} b_{i}{ }^{\prime} k j\right. \\
\\
+\frac{1}{J L} \sum_{j \ell}\left(e_{i k \ell j}-e_{i}{ }^{\prime} k \ell\right.
\end{array}\right]^{2} \\
& V\left(\hat{Y}_{i / k}\right)=\frac{2}{J L}\left(L \sigma_{t b}^{2}+L \sigma_{t t^{\prime} b}^{2}+\sigma^{2}\right)
\end{aligned}
$$

Multiplicando-se e dividindo-se por $k$, não se altera o valor da $V\left(\widehat{Y}_{i / k}\right)$ e ainda, agrupando-se conve nientemente, tem-se:

$$
V\left(\bar{Y}_{i / k}\right)=\frac{2}{J K L}\left[\begin{array}{c}
\left(K L \sigma_{t b}^{2}+L \sigma_{t t^{\prime} b}^{2}+\sigma^{2}\right)+ \\
+(K-1)\left(L \sigma_{t t^{\prime} b}^{2}+\sigma^{2}\right)
\end{array}\right]
$$

esua estimativa será:

$$
\overline{\mathrm{V}}\left(\hat{\mathrm{Y}}_{\mathrm{i} / \mathrm{k}}\right)=\frac{2}{\mathrm{JKL}}[\mathrm{QMR}(\mathrm{a})+(\mathrm{K}-1) \mathrm{QMR}(\mathrm{c})],
$$

que é um resultado concordante com aqueles encontrados em CHAKRABART I (1962), COCHRAN e COX (1976), ZIMMERMANN (1973) para a mesma variância quando o ensaio em faixas com dois trat amentos é delineado em blocos casualizados.

A variância do contraste assim estimado terá n' graus de liberdade, que, de acordo com a f́örmula de SATTERTHWATE (1946), serà: 


$$
n^{\prime}=\frac{[Q M \operatorname{Residuo}(a)+(K-1) Q M \operatorname{Residuo}(c)]^{2}}{\frac{[Q M \operatorname{Residuo}(a)]^{2}}{(I-1)(J-1)}+\frac{(K-1)^{2}[Q M \operatorname{Residuo}(c)]^{2}}{(I-1)(J-1)(K-1)}}
$$

e) Diferença entre duas médias de tratamentos $T^{\prime}$ dentrode um mesmo nível de tratamento $T$ :

$$
\hat{\mathrm{Y}}_{\mathrm{k} / \mathrm{i}}=\hat{\mathrm{m}}_{\mathrm{ik}}-\hat{\mathrm{m}}_{\mathrm{ik}},
$$

Por processos idēnticos ao usado nos itens an teriores, tem-se:

$$
\begin{aligned}
& V\left(\hat{Y}_{k / i}\right)=\frac{2}{J L}\left(L \sigma_{t}^{2} b+L \sigma_{t t^{\prime} b}^{2}+\sigma^{2}\right) . \\
& \text { Multiplicando-se e dividindo-se por I, e ain }
\end{aligned}
$$
da agrupando-se convenientemente:

$$
V\left(\hat{Y}_{k / \dot{i}}\right)=\frac{2}{I J L}\left[\begin{array}{l}
\left(I L \sigma_{t}^{2} b^{\prime}+L \sigma_{t t^{\prime} b}^{2}+\sigma^{2}\right)+ \\
+(I-1)\left(L \sigma_{t t^{\prime} b}^{2}+\sigma^{2}\right)
\end{array}\right]
$$

e sua estimativa

$$
\hat{\mathrm{V}}\left(\hat{\mathrm{Y}}_{\mathrm{k} / \mathrm{i}}\right)=\frac{2}{\mathrm{IJL}}[\mathrm{QMR}(\mathrm{b})+(\mathrm{I}-1) \mathrm{QMR}(\mathrm{c})],
$$

que é um resultado concordante com aqueles encontrados em CHAKRABART I (1962), COCHRAN e COX (1976) e ZIMMERMANN (1973) para a mesma variancia quando o ensaio em faixas com dois tratamentos é delineado em blocos casualizados. 
A variāncia do contraste assim estimado terá n' graus de liberdade, que de acordo com a fórmula de SAT TERTHWAITE (1946) serä:

$$
n^{\prime}=\frac{[Q M \operatorname{Residuo}(b)+(I-1) Q M \operatorname{Residuo}(c)]^{2}}{\frac{[Q M \operatorname{Residuo}(b)]^{2}}{(J-1)(K-1)}+\frac{(I-1)^{2}[Q M \operatorname{Residuo}(c)]^{2}}{(I-1)(J-1)(K-1)}}
$$

E) Diferença entre duas médias de tratamentos T dentro de um mesmo nivel de tratamento $T *$ :

$$
\hat{\mathrm{Y}}_{i / \ell}=\overline{\mathrm{m}}_{\mathrm{i \ell}}-\overline{\mathrm{m}}_{i \cdot \ell} \text {. }
$$

Por processos idénticos ao usado nos itens an teriores, tem_se:

$$
V\left(\hat{Y}_{i / \ell}\right)=\frac{2}{J K}\left(K \sigma_{t b}^{2}+\sigma_{t t^{\prime} b}^{2}+\sigma^{2}\right),
$$

multiplicando-se e dividindo-se por L e agrupando-se convenientemente:

$$
V\left(\hat{Y}_{i / \ell}\right)=\frac{2}{J K L}\left[K L \sigma_{t b}^{2}+L \sigma_{t t}^{2} b+\sigma^{2}+(L-1) \sigma^{2}\right]
$$

esua estimativa:

$$
\hat{\mathrm{V}}\left(\hat{\mathrm{Y}}_{\mathrm{i} / \ell}\right)=\frac{2}{\mathrm{JKL}}[\mathrm{QMR}(\mathrm{a})+(\mathrm{L}-1) \operatorname{QMR}(\mathrm{d})]
$$

com n' graus de liberdade, onde: 


$$
\mathrm{n}^{\prime}=\frac{[\mathrm{QM} \operatorname{Residuo}(\mathrm{a})+(\mathrm{L}-1) \mathrm{QM} \operatorname{Residuo}(\mathrm{d})]^{2}}{\frac{[\mathrm{QM} \operatorname{Residuo}(\mathrm{a})]^{2}}{(\mathrm{I}-1)(\mathrm{J}-1)}+\frac{(\mathrm{L}-1)^{2}[\mathrm{QM} \operatorname{Residuo}(\mathrm{d})]^{2}}{\operatorname{IK}(\mathrm{J}-1)(\mathrm{L}-1)}}
$$

g) Diferença entre duas médias de tratamentos $T$ * dentro de um mesmo nivel de tratamento $T$ :

$$
\hat{\mathrm{Y}}_{\ell / i}=\hat{\mathrm{m}}_{\mathrm{i} \ell}-\hat{\mathrm{m}}_{\mathrm{i} \ell} \cdot
$$$$
\text { Do mésmo modo tem-se }
$$$$
V\left(Q_{\ell / i}\right)=\frac{2}{J K} \sigma^{2}
$$

e sua estimativa serä:

$$
\vec{\nabla}\left(\hat{Y}_{\ell / i}\right)=\frac{2}{J K}[Q M, R(d)]
$$

h) Diferença entre duas médias de tratamentos $T^{\prime}$ dentro de um mesmo nivel de tratamento $T *$ :

$$
\hat{\mathrm{y}}_{\mathrm{k} / \ell}=\hat{\mathrm{m}}_{\mathrm{k} \ell}-\hat{\mathrm{m}}_{\mathrm{k} \cdot \ell}
$$

De forma idēntica às adotadas anteriormente,

t em-s e :

$$
V\left(\hat{Q}_{k / l}\right)=\frac{2}{I J}\left(I \sigma_{t^{\prime} b}^{2}+\sigma_{t t^{\prime} b}^{2}+\sigma^{2}\right)
$$


Multiplicando-se e dividindo-se por L e agrü pando-se convenientemente, tem-se:

$$
V\left(\varphi_{k / \ell}\right)=\frac{2}{I J L}\left[\left(I L \sigma_{t}^{2} b+L \sigma_{t t}^{2} b+\sigma^{2}\right)+(L-1) \sigma^{2}\right]
$$

e sua estimativa:

$$
\widehat{V}\left(\hat{\mathrm{Y}}_{\mathrm{k} / \ell}\right)=\frac{2}{\mathrm{IJL}}[\mathrm{QMR}(\mathrm{b})+(\mathrm{L}-1) \mathrm{QMR}(\mathrm{d})],
$$

com n' graus de liberdade, onde:

$$
n^{\prime}=\frac{[Q M \operatorname{Residuo}(b)+(L-1) Q M \operatorname{Residuo}(d)]^{2}}{\frac{[Q M \operatorname{Residuo}(b)]^{2}}{(K-1)(J-1)}+\frac{(L-1)^{2}[Q M \operatorname{Residuo}(d)]^{2}}{I K(J-1)(L-1)}}
$$

i) Diferença entre duas médias de tratamento:s T* dentro de um mesmo nivel de tratamento $T^{\prime}$ :

$$
\hat{\mathrm{Y}}_{\ell / \mathrm{k}}=\widehat{\mathrm{m}}_{\mathrm{k} \ell}-\hat{\mathrm{m}}_{\mathrm{k} \ell} \text {, }
$$

Por processos idēnticos aos usados anteriormente, tem-se:

$$
V\left(\hat{Y}_{\ell / k}\right)=\frac{2}{I J} \sigma^{2}
$$

e sua estimativa:

$$
\vec{\nabla}\left(\hat{Y}_{\ell / k}\right)=\frac{2}{I J}[Q M R(d)]
$$


j) Diferença entre duas médias de tratamentos T, dentro de uma mesma combinação $T^{\prime} T^{*}$ :

$$
\hat{\mathrm{Y}}_{i / k \ell}=\hat{\mathrm{m}}_{\mathrm{ik} \ell}-\hat{\mathrm{m}}_{\mathrm{i}} \mathrm{k}_{\mathrm{k} \ell} \text {. }
$$

A variāncia do contraste dado pela fórmula:

$$
V\left(\hat{Y}_{i / k \ell}\right)=E\left[\hat{Y}_{i / k \ell}-E\left(\hat{Y}_{i} \cdot k \ell\right)\right]^{2}
$$

है gual a:

$$
V\left(\bar{Y}_{i / k \ell}\right)=\frac{2}{J}\left(\sigma_{t b}^{2}+\sigma_{t t^{\prime} b}^{2}+\sigma^{2}\right)
$$

Multiplicando-se e dividindo-se por KL e agrupando-se convenientemente, vem:

$$
V\left(\hat{Y}_{i / k \ell}\right)=\frac{2}{J K L}\left[\begin{array}{l}
\left(K L \sigma_{t b}^{2}+L \sigma_{t t^{\prime} b}^{2}+\sigma^{2}\right)+ \\
+(K-1)\left(L \sigma_{t t^{\prime} b}^{2}+\sigma^{2}\right)+K(L-1) \sigma^{2}
\end{array}\right]
$$

cuja estimativa è dada por:

$$
\mathrm{V}\left(\hat{\mathrm{Y}}_{\mathrm{i} / \mathrm{k} \ell}\right)=\frac{2}{\mathrm{JKL}}[\mathrm{QMR}(\mathrm{a})+(\mathrm{K}-1) \mathrm{QMR}(\mathrm{c})+\mathrm{K}(\mathrm{L}-1) \mathrm{QMR}(\mathrm{d})],
$$

com n' graus de liberdade, onde:

$$
\mathrm{n}^{\prime}=\frac{[\mathrm{QM} \operatorname{Residuo}(\mathrm{a})+(\mathrm{K}-1) \mathrm{QM} \operatorname{Residuo}(\mathrm{c})+\mathrm{K}(\mathrm{L}-1) \mathrm{QM} \operatorname{Residuo}(\mathrm{d})]^{2}}{\frac{[\mathrm{QM} \operatorname{Residuo}(\mathrm{a})]^{2}}{(\mathrm{I}-1)(\mathrm{J}-1)}+\frac{(\mathrm{K}-1)^{2}[\mathrm{QM} \operatorname{Residuo}(\mathrm{c})]^{2}}{(\mathrm{I}-1)(\mathrm{J}-1)(\mathrm{K}-1)}+\frac{\mathrm{K}^{2}(\mathrm{~L}-1)^{2}[\mathrm{QM} \operatorname{Residuo}(\mathrm{d})]^{2}}{\mathrm{IK}(\mathrm{J}-1)(\mathrm{L}-1)}}
$$


k) Diferença entre duas médias de tratamentos $T^{\prime}$, dentro de uma mesma combinação TT*:

$$
\hat{P}_{k / i \ell}=\hat{m}_{i k \ell}-\hat{m}_{i k} \ell \text {. }
$$

A variāncia do contraste em questão serä:

$$
V\left(Q_{k / i \ell}\right)=\frac{2}{J}\left(\sigma_{t}^{2}{ }^{\prime} b+\sigma_{t t^{\prime} b}^{2}+\sigma^{2}\right)
$$

$$
\text { Multiplicando-se e dividindo-se por IL, e }
$$

ainda agrupando-se convenientemente, tem-se:

$$
V\left(\hat{Y}_{k / i l}\right)=\frac{2}{I J L}\left\{\begin{array}{l}
\left(I L \sigma_{t}^{2} b+L \sigma_{t t^{\prime} b}^{2}+\sigma^{2}\right)+ \\
+\left[(I-1) L \sigma_{t t^{\prime} b}^{2}+\sigma^{2}\right]+ \\
+\left[I(L-1) \sigma^{2}\right]
\end{array}\right]>
$$

e sua estimativa:

$$
\widehat{V}\left(\widehat{Y}_{k} / i_{\ell}\right)=\frac{2}{I J L}[\mathrm{QMR}(\mathrm{b})+(\mathrm{I}-1) \mathrm{QMR}(\mathrm{c})+\mathrm{I}(\mathrm{L}-1) \mathrm{QMR}(\mathrm{d})],
$$

com n' graus de liberdade, onde:

$$
n^{\prime}=\frac{[Q M \operatorname{Residuo}(b)+(I-1) Q M \operatorname{Residuo}(c)+I(L-1) Q M \operatorname{Residuo}(d)]^{2}}{\frac{[Q M \operatorname{Residuo}(b)]^{2}}{(\mathrm{~K}-1)(\mathrm{J}-1)}+\frac{(\mathrm{I}-1)^{2}[\mathrm{QM} \operatorname{Residuo}(\mathrm{c})]^{2}}{(\mathrm{I}-1)(\mathrm{J}-1)(\mathrm{K}-1)}+\frac{\mathrm{I}^{2}(\mathrm{~L}-1)^{2}[\mathrm{QM} \operatorname{Residuo}(\mathrm{d})]^{2}}{\operatorname{IK}(\mathrm{J}-1)(\mathrm{L}-1)}}
$$


1) Diferença entre duas médias de tratamentos T* dentrode uma mesma combinação T'T:

$$
\begin{gathered}
\hat{\mathrm{Y}}_{\ell / \mathrm{ik}}=\hat{\mathrm{m}}_{\mathrm{ik} \ell}-\hat{\mathrm{m}}_{\mathrm{ik} \ell} \\
\text { Para o contraste em estudo, tem-se: } \\
\mathrm{V}\left(\hat{\mathrm{Y}}_{\ell / \mathrm{ik}}\right)=\frac{2}{\mathrm{~J}} \sigma^{2}
\end{gathered}
$$

e sua estimativa será:

$$
\widehat{V}\left(\hat{Y}_{\ell / i k}\right)=\frac{2}{J}[Q M R(d)] .
$$

\section{5 - Comparações Múltiplas}

Visando à estruturação dos contrastes entre duas médias de tratamentos, foram estudados doze casos que constituem os de maior interesse do ponto de vista de aplicação. Em cada caso foi estabelecido o contraste propriamente dito, determinada a sua variāncia e, quando ca-

bivel, deduzido o nümero de graus de liberdade pela fórmu 1a de SATTERTHWATE (1946).

Com estes elementos é possivel utilizar-se qualquer dos métodos clässicos de comparações mültiplas, desde que estejam resguardadas as pressuposições do mode10. Nesse estudo optou-se pelo critério de Tukey, por sua 
versatilidade e aplicabilidade.

Assim, com base na diferenca mínima significativa, dada por $\Delta=q \sqrt{1 / 2 \hat{Q}(\bar{Y})}$, onde q é o valor da am plitude total estudentizada e $\nabla(\bar{Y})$ é a estimativa da variāncia da estimativa do contraste em questão, foram obti d as :

a) Para contrastes entre médias de tratamento T:

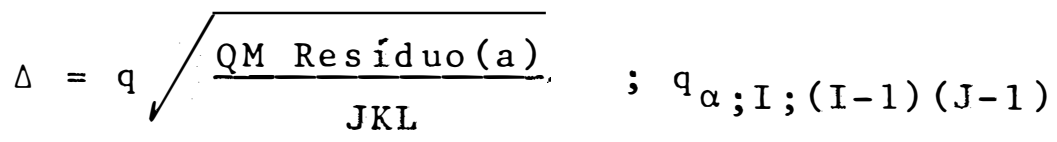

b) Para contrastes entre médias de tratamentos T':

$$
\Delta=\mathrm{q} \sqrt{\frac{\mathrm{QMRR} \operatorname{Residuo(b)}}{\mathrm{IJL}}} ; \mathrm{q}_{\alpha ; \mathrm{K} ;(\mathrm{K}-1)(\mathrm{J}-1)} \text {. }
$$

c) Para contrastes entre médias de tratamentos $\mathrm{T}^{*}$ :

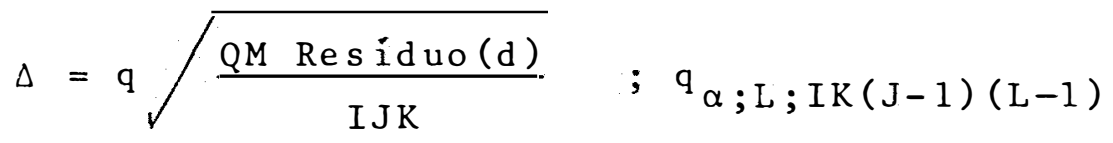

d) Para contrastes entre médias de tratamentos $T$ dentro de um mesmo nivel de tratamento $\mathrm{T}^{\prime}$ :

$$
\Delta=q \sqrt{\frac{\mathrm{QMResiduo}(\mathrm{a})+(\mathrm{K}-1) \mathrm{QM} \operatorname{Residuo}(\mathrm{c})}{\mathrm{JKL}}} ; \mathrm{q}_{\alpha ; I ; \mathrm{n}^{\prime}}
$$

onde $n^{\prime}$ é obtido conforme 3.4.1.d. 
e) Para contrastes entre médias de tratamentos $T^{\prime}$ dentrode um mesmo nivel de T:

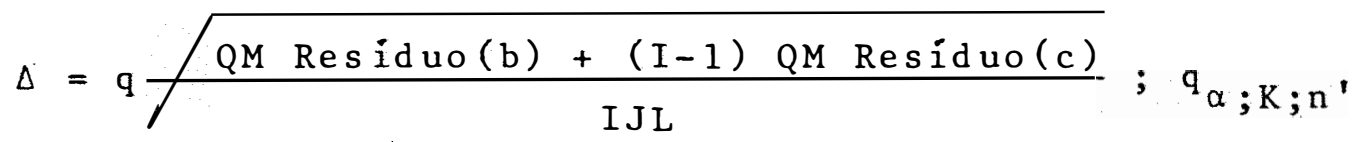

onde $n^{\prime}$ è obtido conforme 3.4.1.e.

E) Para contrastes entre médias de tratamentos $T$, dentrode um mesmo nivel de tratamento $\mathrm{T} *$ :

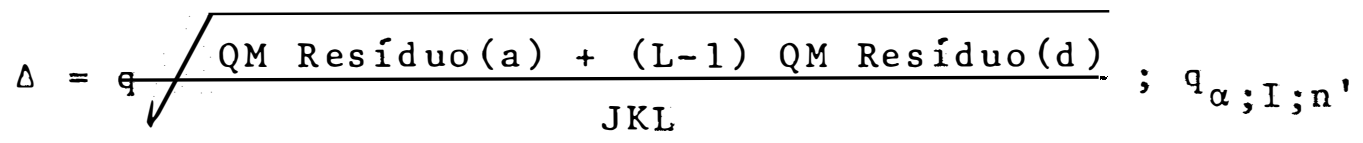

onde $n^{\prime}$ é obtido conforme 3.4.1.f.

g) Para contrastes entre mëdias de tratamentos $T$ * dentrode um mesmo nivel de tratamento T:

$$
\Delta=q \sqrt{\frac{Q M \operatorname{Residuo}(\mathrm{d})}{\mathrm{JK}}} ; \mathrm{q}_{\alpha ; L ; I K(J-1)(\mathrm{L}-1)}
$$

h) Para contrastes entre médias de tratamentos $T^{\prime}$ dentrode um mesmo nivel de tratamento $\mathrm{T} *$ :

$$
\Delta=\sqrt{\frac{Q M \operatorname{Residuo}(b)+(L-1) Q M \operatorname{Residuo}(d)}{\text { IJL }}} ; q_{\alpha ; K ; n} \text { ' }
$$

onde $n^{\prime}$ é obtido conforme 3.4.1.h. 
i) Para contrastes entre mëdias de tratamentos $T$ * dentrode um mesmo nivel. de tratamento T':

$$
\Delta = \mathrm { q } \longdiv { \frac { \mathrm { QM } \operatorname { R e s i d u o ( d ) } } { \mathrm { IJ } } } \quad ; \mathrm{q}_{\alpha ; \mathrm{L} ; \mathrm{IK}(\mathrm{J}-1)(\mathrm{L}-1)}
$$

j) Para contrastes entre mëdias de tratamentos T dentro de uma mesma combinação $T^{\prime} T$ * :

$\Delta=q \sqrt{\frac{Q M \operatorname{Residuo}(\mathrm{a})+(\mathrm{K}-1) \mathrm{QM} \operatorname{Residuo}(\mathrm{c})+\mathrm{K}(\mathrm{L}-1) \mathrm{QM} \operatorname{Residuo}(\mathrm{d})}{\mathrm{JKL}}}$

$q_{\alpha ; I ; n^{\prime}}$, onde $n^{\prime} e^{-}$obtido conforme $3.4 .1 . j$.

k) Para çontrastes entre médias de tratamentos $T^{\prime}$ dentrode uma mesma combinação T T* :

$\Delta=q \sqrt{\frac{Q M \operatorname{Residuo}(b)+(I-1) Q M \operatorname{Residuo}(c)+\mathrm{I}(\mathrm{L}-1) \mathrm{QM} \operatorname{Residuo}(\mathrm{d})}{\mathrm{IJL}}}$

$\AA_{\alpha ; K ; n^{\prime}}$; onde $n^{\prime}$ è obtido conforme 3.4.1.k.

1) Para contrastes entre médias de tratamentos $\mathrm{T}$ * dentro de uma mesma combinação T'T:

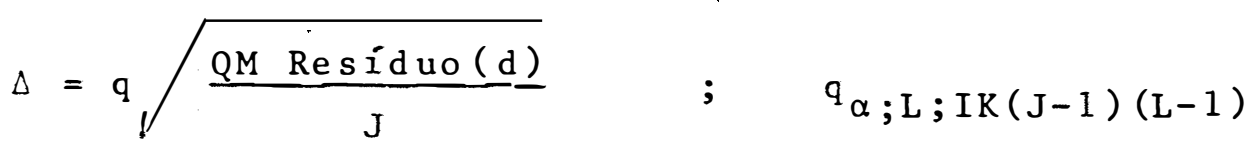


74.

4. UM EXEMPLO DE APLICACAOO

Com o objetivo de ilustrar as técnicas sugeridas nas deduções do item 3 , apresenta-se a seguir um exemplo de aplicação, onde foram considerados:

$\mathrm{T}=$ duas épocas de plantio: 11/09/78 e 31/10/78;

$\mathrm{T}^{\prime}=$ très variedades de cana-de-açücar: CB 41-76, IAC 51-205 e NA 56-79;

$\mathrm{T}^{*}=$ nümero de gemas por colmo: uma e três.

o experimento foi conduzido em cinco repetiçoes, em blocos ao acaso, considerando-se $T^{2} T^{\prime}$ em faixas e T* como subtratamento em cada combinação TT', conforme ilustrado no esquema seguinte: 


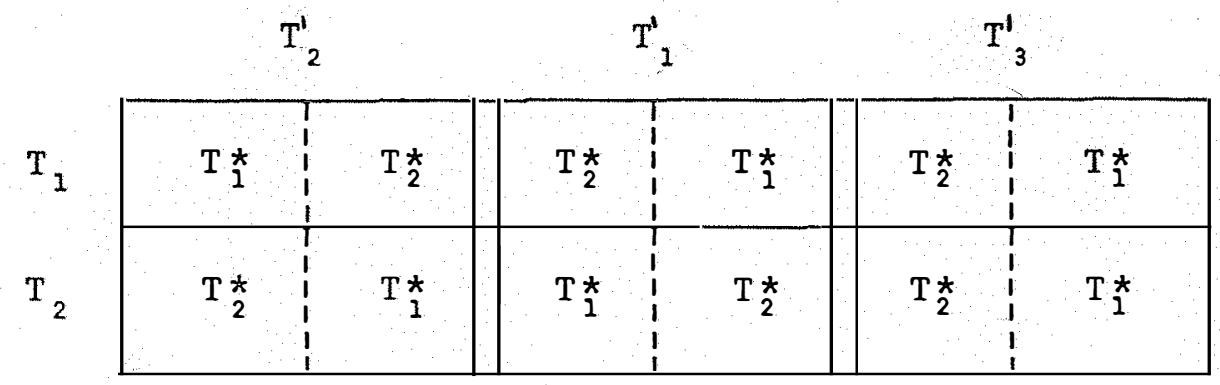

os pesos médios dos colmos, em kg/parcela, constam do Quadro 10 .

Quadro 10 - Peso médio dos colmos, em kg por parcela.

\begin{tabular}{|c|c|c|c|c|c|c|c|}
\hline VARIEDADES & EPOCAS & GEMAS & I & II & III & IV & $\mathrm{v}$ \\
\hline \multirow{4}{*}{ CB $41-76$} & \multirow{2}{*}{$1 a$} & 1 & 272,70 & 318,40 & 316,20 & 246,00 & 275,00 \\
\hline & & 3 & 324,50 & 320,10 & 297,30 & 331,20 & 296,10 \\
\hline & \multirow[t]{2}{*}{$2 a$} & 1 & 231,50 & 229,00 & 201,00 & 192,20 & 205,50 \\
\hline & & 3 & 196,00 & 232,00 & 232,00 & 273,20 & 244,00 \\
\hline \multirow{4}{*}{ IAC $51 / 205$} & \multirow{2}{*}{19} & 1 & $180,50$. & 195,90 & 220,90 & 186,50 & 229,50 \\
\hline & & 3 & 248,70 & 245,00 & 217,50 & 249,50 & 255,00 \\
\hline & \multirow{2}{*}{$2 a$} & 1 & 155,00 & 188,00 & 180,50 & 181,50 & 128,00 \\
\hline & & 3 & 218,70 & 209,70 & 202,00 & 222,20 & 225,50 \\
\hline \multirow{4}{*}{ NA $56-79$} & \multirow[t]{2}{*}{$1 a$} & 1 & 144,70 & 98,50 & 146,10 & 79,00 & 100,50 \\
\hline & & 3 & 245,80 & 265,40 & $227 ; 80$ & 260,80 & 228,50 \\
\hline & \multirow[b]{2}{*}{$2 a$} & 1 & 105,50 & 127,20 & 132,00 & 184,20 & 156,50 \\
\hline & & 3 & 193,00 & 192,00 & 221,00 & 216,00 & 165,30 \\
\hline
\end{tabular}

Conforme as regras deduzidas em 3.3 .3 , obte ve-se a análise de variāncia que consta do Quadro 11. 
Quadro 11 - Anälise de variāncia dos pesos médios dos col mos .

\begin{tabular}{lcrrr}
\hline Causas da Variação & G.L. & S.Q. & Q.M. & F \\
\hline Blocos & 4 & $1.036,0876$ & 259,0219 & - \\
Épocas & 1 & $23.340,5926$ & $23.340,5926$ & $35,37 * *$ \\
Residuo (a) & 4 & $2.639,2424$ & 659,8106 & - \\
Variedades & 2 & $77.686,2223$ & $38.843,1112$ & $207,67 * *$ \\
Residuo (b) & 8 & $1.496,3094$ & 187,0387 & - \\
Epoca x Variedade & 2 & $11.221,0564$ & $5.610,5282$ & $14,67 * *$ \\
Residuo (c) & 8 & $3.060,3286$ & 382,5411 & - \\
Gemas & 1 & $45.254,0806$ & $45.254,0806$ & $63,75 * *$ \\
Época x Gema & 1 & $2.133,6808$ & $2.133,6808$ & 3,01 \\
Variedade x Gema & 2 & $12.421,8904$ & $6.210,9452$ & $8,75 * *$ \\
Epoca x Variedade x Gema & 2 & $5.112,7922$ & $2.556,3961$ & 3,60 \\
Residuo (d) & 24 & $17.037,2760$ & 709,8865 & - \\
\hline Total & 59 & $202.439,5593$ & & \\
\hline
\end{tabular}

Através dos procedimentos descritos em (3.4.2) foram obtidas as seguintes diferencas minimas significativas (DMS), relativas ao método de Tukey. 


\begin{abstract}
Quadro 12 - Contrastes entre duas médias de tratamentos, suas respectivas variāncias e a diferença minima significativa (DMS) calculada pelo método de Tukey, à taxa de 0,05 de probabilidade.
\end{abstract}

\begin{tabular}{|c|c|c|c|}
\hline CONTRASTE & $n^{\prime}$ & $\nabla(\bar{Y})$ & DMS \\
\hline$\hat{Y}_{i}$ & 4 & 43,9874 & 18,43 \\
\hline$\hat{\mathrm{Y}}_{\mathrm{k}}$ & 8 & 18,7039 & 12,36 \\
\hline$\hat{Y}_{\ell}$ & 24 & 47,3258 & 14,20 \\
\hline$\hat{Y}_{i / k}$ & 11 & 94,9929 & 21,43 \\
\hline$\widehat{\mathrm{Y}}_{\mathrm{k} / \mathrm{i}}$ & 14 & 56,9580 & 19,75 \\
\hline $\bar{Y}_{i / \ell}$ & 14 & 91,3131 & 20,47 \\
\hline$\hat{\mathrm{Y}}_{\ell / \mathrm{i}}$ & 24 & 94,6515 & 20,09 \\
\hline$\hat{\mathrm{Y}}_{\mathrm{k} / \ell}$ & 31 & 89,6925 & 23,34 \\
\hline$\widehat{\mathrm{Y}}_{\ell / \mathrm{k}}$ & 24 & 141,9773 & 24,60 \\
\hline$\widehat{\mathrm{Y}}_{\mathrm{i} / \mathrm{kl}}$ & 34 & 236,9702 & 31,33 \\
\hline$\widehat{Y}_{k / i l}$ & 37 & 198,9353 & 34,47 \\
\hline$\hat{\mathrm{Y}}_{\ell / \mathrm{ik}}$ & 24 & 283,9546 & 34,79 \\
\hline
\end{tabular}


78 .

\section{CONCLUSOES}

De um modo geral, concluiu-se que:

5.1 - 0 modelo linear adequado para a anàlise de ensaios em faixas com subparcelas subdivididas, pọ de ser obtido através da inclusão dos parâmetros relativos ao tratamento secundàrio, no modelo tradicional dos experimentos em faixas.

5.2 - Na anālise de variância de ensaios em faixas com subparcelas subdivididas, pode-se identificar: duas partes distintas. A primeira envolvendo os trata mentos principais numa estrutura anäloga ao esquema usual dos experimentos em faixas e a segun da constituida do tratamento secundàrio e suas interações com os tratamentos principais. 
5.3 - Os componentes de variāncia confirmaram a analogia referida na conclusão anterior, no tocante aos residuos apropriados para os testes adequados a cada tipo de tratamento e suas interações.

5.4 - As variâncias dos contrastes de interesse para uso nos critérios de comparações mültiplas se adequaram a cada parte anteriormente citada da es trutura da anälise de variància, isto é, onde en volveu os tratamentos principais e sua interaça, como experimentos em faixas e onde envolveuo tra tamento secundário e suas interaçoes com os prin cipais, como experimento em parcelas subsubdivididas. 
80 .

\title{
6. REFERENCIAS BIBLIOGRÄFICAS
}

\author{
ANDERSON, R.L. e T.A. BANCROFT, 1952. Statistical Theory. \\ in Research. McGraw-Hill, Nova York, 399 pp. \\ CHAKRABARTI, M.C., 1962. Mathematics of Design and Ana- \\ lysis of Experiments. Asia Publishing House, Londres, \\ 120 pp. \\ COCHRAN, W.G. e G.M. COX, 1976. Diseños Experimentales. \\ 3a ed. Trillas, Mexico, 661 pp. \\ FEDERER, W.T., 1955. Experimental Design. 1 ạ ed., McMil \\ lan Co., Nova York, 544 p . \\ GOULDEN, C.H., 1939. Methods of Statistical Analysis. \\ Wiley, Nova York, 151 pp. \\ HEDAYETULLAH, S.; S. SEN e K.R. NAIR, 1944. Influence of \\ Dates of Planting and Spacings on Some Winter Varieties \\ of Rice. Indian J. Agr. Sci., 14: 248-259.
}


KEMPTHORNE, 0., 1952. The Design and Analysis of Experiments. 1 a ed. Wiley, Nova York, 631 pp.

LOMA, J.L. de La, 1966. Experimentaciōn Agrícola, 2 a ed. México. Union Tipográfica Editorial Hispano Americana, 495 pp.

NAIR, K.R., 1944. Calculation of the standard error and tests of significance of different types of treatment comparisons in split-plot and steep arrangements of fields experiments. Indian J. Agr. Sci., 14: 315-319.

NOGUEIRA, I.R., 1962. Emprego do cálculo de matrizes na dedução de importante fórmula da análise estatistica . Boletim Técnico-Cientifico, ESALQ/USP, 1: 1-8.

PANSE, V.G. e P.V. SUKHATME, 1963. Mẻtodos Estadisticos para Investigadores Agrícolas. 2a ed. México, Fondo de Cultura Econômica, 359 pp.

PIMENTEL GOMES, F., 1966. Componentes de Variāncia. Apos tila mimeografada. Escola Superior de Agricultura "Luiz de Queiroz" - USP, Piracicaba.

PIMENTEL GOMES, F., 1976. Curso de Estatistica Experimenta1. 6a ed. Nobe1, Piracicaba, 430 pp.

SATTERTHWATtE, F.E., 1946. An aproximate distribution of estimates of variance components. Biometrics, 2: 110-114 . 
82.

ZIMMERMANN, F.J.P., 1973. Anälise de um experimento de adubação e calagem em milho, executado num delineamento em faixa durante oito anos no mesmo local. Piracicaba, ESALQ/USP. 93 pp. [Dissertação de Mestrado]. 